TRANSACTIONS OF THE

AMERICAN MATHEMATICAL SOCIETY

Volume 355, Number 2, Pages 787-811

S 0002-9947(02)03134-3

Article electronically published on October 2, 2002

\title{
QUASICONFORMAL GROUPS, PATTERSON-SULLIVAN THEORY, AND LOCAL ANALYSIS OF LIMIT SETS
}

\author{
PETRA BONFERT-TAYLOR AND EDWARD C. TAYLOR
}

\begin{abstract}
We extend the part of Patterson-Sullivan theory to discrete quasiconformal groups that relates the exponent of convergence of the Poincaré series to the Hausdorff dimension of the limit set. In doing so we define new bi-Lipschitz invariants that localize both the exponent of convergence and the Hausdorff dimension. We find these invariants help to expose and explain the discrepancy between the conformal and quasiconformal setting of PattersonSullivan theory.
\end{abstract}

\section{INTRODUCTION}

Let $G$ be a quasiconformal Fuchsian group, namely a discrete group of uniformly $K$-quasiconformal mappings, each of which preserves the closed unit ball $\overline{\mathbb{B}^{n}}$. In this paper we explore the connections between the discrete action of the group on $\mathbb{S}^{n-1}=\partial \mathbb{B}^{n}$ and its discontinuous action on $\mathbb{B}^{n}$. Our techniques involve analyzing the relationship between the asymptotic rate of accumulation of orbit points, as measured via the exponent of convergence $\delta(G)$ of the Poincaré series of the group, and the Hausdorff dimension of the limit set $L(G) \subset \mathbb{S}^{n-1}$.

In the setting of Kleinian groups, i.e., those quasiconformal Fuchsian groups consisting entirely of Möbius transformations, there is a well-developed theory that connects the geometric action of the group on hyperbolic space $\left(\mathbb{B}^{n}, \rho\right)$ to its conformal action on the sphere at infinity. If $\Gamma$ is a Kleinian group with more than two limit points in its limit set, then it is a result (separately) of Patterson, Sullivan, and Bishop and Jones that the exponent of convergence of the Poincaré series equals the Hausdorff dimension of a dense subset of the limit set. In the case where $\Gamma$ is convex co-compact, the Patterson-Sullivan proof is as follows: One first shows the existence of a finite and positive measure that is supported on the limit set, that scales correctly under the group action, and that behaves locally like the Hausdorff measure of dimension $\delta(\Gamma)$. One then uses the ergodic properties of the isometric action of the group to conclude that this measure is in fact a constant multiple of the Hausdorff measure.

A quasiconformal Fuchsian group (not Kleinian), while acting discontinuously on $\mathbb{B}^{n}$, no longer acts isometrically on $\left(\mathbb{B}^{n}, \rho\right)$. Our goal is to explore how much

Received by the editors September 15, 2000 and, in revised form, May 14, 2002.

2000 Mathematics Subject Classification. Primary 30C65, Secondary 30F40, 30F45.

Key words and phrases. Kleinian groups, discrete quasiconformal groups, Patterson-Sullivan measure, exponent of convergence, Hausdorff dimension.

The first author was supported in part by NSF grant 0070335 .

The second author was supported in part by an NSF Postdoctoral Fellowship.

(C)2002 American Mathematical Society 
of Patterson-Sullivan theory remains valid in this setting. Somewhat surprisingly, we recover a fair amount of the original theory. Some of our principle results are summarized below:

Results: Let $G$ be a discrete non-elementary quasiconformal Fuchsian group acting on $\mathbb{B}^{n}$.

(1) For each $x \in \mathbb{B}^{n}$, the Patterson-Sullivan construction yields a measure class of finite and positive measures with support in the limit set (Theorem 4.3).

(2) Crucially, these measures do not typically satisfy a certain transformation property characteristic of Patterson-Sullivan measures supported on the limit sets of Kleinian groups. Conversely, we show that this transformation property supplies a sufficient condition for quasiconformal Fuchsian groups to be Kleinian (Theorem 5.1).

(3) The support of each measure in the measure class is typically a proper subset of the limit set (Theorem 7.1). To isolate the support, one "localizes" the definition of the exponent of convergence. We find that the support is contained in the set on which the local exponent of convergence is maximized. We also show that these new invariants are local bi-Lipschitz invariants in the Euclidean metric (Theorem 6.10). The usefulness of this observation is that every element of a quasiconformal Fuchsian group is almost everywhere locally bi-Lipschitz (whereas Möbius transformations are everywhere bi-Lipschitz). This helps to reveal (see Example 8.1) how PattersonSullivan theory in the quasiconformal setting differs in other ways as well from the theory in the Möbius setting.

(4) We show that the local exponent of convergence is an upper bound on the local Hausdorff dimension of the conical limit set (Theorem 6.7). Thus we will be able to recover $\operatorname{dim}\left(L_{c}(G)\right) \leq \delta(G)$ (Theorem 6.8). We provide an example of a quasiconformal Fuchsian group having a finite-sided fundamental polyhedron such that $\operatorname{dim}\left(L_{c}(G)\right)<\delta(G)$, and $G$ 's PattersonSullivan measures have atoms (Example 8.1).

(5) We provide sufficient geometric conditions for a quasiconformal action on $\mathbb{B}^{n}$ so that $\operatorname{dim}\left(L_{c}(G)\right)=\delta(G)$ (Theorem 9.1). Under the same assumptions, we show that the localized quantities are similarly well-behaved.

We proceed as follows: Section 2 contains useful facts from the theory of quasiconformal groups. In Section 3 we provide some basic results on the exponent of convergence. In Section 4 we outline the Patterson-Sullivan construction in the quasiconformal setting. In Section 5 we analyze the transformation property characteristic of Patterson-Sullivan measures supported on the limit sets of Kleinian groups. In Section [6] we define the tools necessary in the local analysis of limit sets of quasiconformal groups and prove some of their fundamental properties. Section 7 contains the result that shows that the Patterson-Sullivan measure is supported on the subset of the limit set where the local exponent is maximal. Finally, in Section 8 we build examples, and in Section 9 we give a sufficient condition on the behavior of Patterson-Sullivan measure under the action of the group so that the Hausdorff dimension of the conical limit set equals the exponent of convergence. 


\section{Quasiconformal Group Basics}

A $K$-quasiconformal group $G$ acting on $\overline{\mathbb{R}^{n}}$ is a group of mappings, each of which is a $K$-quasiconformal homeomorphism of $\overline{\mathbb{R}^{n}}$. Such a group $G$ is discrete if there exists no sequence of elements in the group that converges uniformly on $\overline{\mathbb{R}^{n}}$ to the identity mapping. Here, $\overline{\mathbb{R}^{n}}=\mathbb{R}^{n} \cup\{\infty\}$ is endowed with the chordal metric. We note that a discrete 1-quasiconformal group is a discrete group of Möbius transformations, i.e., a Kleinian group ([28]).

The action of a discrete group $G$ partitions $\overline{\mathbb{R}^{n}}$ into two disjoint sets. The regular set $\Omega(G) \subset \overline{\mathbb{R}^{n}}$ is the largest open set on which $G$ acts discontinuously; the limit set $L(G)$ is the complement of $\Omega(G)$ in $\overline{\mathbb{R}^{n}}$. It is easy to see that $L(G)$ is a closed set; in the case where $L(G)$ contains more than 2 points, then $L(G)$ is a perfect set (and thus uncountable), that is, either nowhere dense or all of $\overline{\mathbb{R}^{n}}$. If $L(G)$ is perfect, then $G$ is called non-elementary. For the basics in the theory of quasiconformal groups see [8]; for discrete Möbius groups $(K=1)$ see [14].

Because we wish to use geometric arguments involving the hyperbolic metric, we will only consider those $K$-quasiconformal groups acting on $\overline{\mathbb{R}^{n}}$ that preserve the unit ball $\mathbb{B}^{n}$. We call such a group $G$ a quasiconformal Fuchsian group, and we label such a $G$ by the symbol "QCF". One way of constructing a QCF group is to conjugate a Kleinian group keeping $\mathbb{B}^{n}$ invariant by a quasiconformal mapping that preserves $\mathbb{B}^{n}$ as well. Not all QCF groups are constructed this way in dimensions $n \geq 3$ (see for example [10], [15] for the case $n=3$ and [12, 23] for the general case). If $G$ is a QCF group, then $\partial \mathbb{B}^{n}$ is also invariant under $G$, and if $G$ is discrete, in addition, then it acts discontinuously in $\mathbb{B}^{n}$, and $L(G) \subset \partial \mathbb{S}^{n-1}$ by Corollary 3.8 in [8]. Note that $L(G)$ is the set of accumulation points (in the chordal metric) of the orbit of any point $y \in \mathbb{B}^{n}$.

Most of our analysis on the limit set consists of working with a geometrically well-behaved dense subset of the limit set. Let $G$ be a discrete QCF group. The conical limit set $L_{c}(G)$ is the set of points $\zeta \in L(G)$ with the following property: there exists an infinite sequence of elements $\left\{g_{k} \in G\right\}$ and a point $z_{0} \in \mathbb{B}^{n}$ so that $\left\{g_{k}\left(z_{0}\right)\right\}$ converges to $\zeta$ within a bounded hyperbolic cone about a geodesic ray ending on $\zeta$. We recall that there is a special class of finitely generated Kleinian groups for which the analytic and geometric theory is most complete. We say that a Kleinian group is geometrically finite if its limit set consists entirely of conical limit points and bounded parabolic limit points (see [6]). Such a geometrically finite group is convex co-compact if its limit set consists only of conical limit points.

\section{The Exponent of Convergence}

In this section we recall basic definitions and results concerning the exponent of convergence of the Poincaré series of a discrete QCF group. Fix a discrete QCF group $G$ acting on $\overline{\mathbb{B}^{n}}$. For a fixed positive real number $s$, and a choice of point $(x, y) \in \mathbb{B}^{n} \times \mathbb{B}^{n}$, the Poincaré series is given by

$$
\Sigma(s, x, y)=\sum_{g \in G} e^{-s \rho(x, g(y))} .
$$

The exponent of convergence is defined to be

$$
\delta(G)=\inf \{s>0 \mid \Sigma(s, x, y)<\infty\}
$$


and does not depend on the choice of $(x, y) \in \mathbb{B}^{n} \times \mathbb{B}^{n}$ as stated in the lemma below. One has various ways of calculating the exponent of convergence; the choice depends on which class of groups one is considering. For our future use, we record the following result (Proposition 2.2 in [5]).

Lemma 3.1. Let $G$ be a discrete $Q C F$ group acting on $\overline{\mathbb{B}^{n}}$. Then for all $x, y \in \mathbb{B}^{n}$,

$$
\begin{aligned}
\delta(G) & =\inf _{s}\{\Sigma(s, x, y)<\infty\}=\inf _{s}\{\Sigma(s, 0,0)<\infty\} \\
& =\inf _{s}\left\{\sum_{g \in G}(1-|g(x)|)^{s}<\infty\right\}=\inf _{s}\left\{\sum_{g \in G}(1-|g(0)|)^{s}<\infty\right\} .
\end{aligned}
$$

There is a well-established upper bound on the exponent of convergence.

Theorem 3.2 (Gehring-Martin [9]). Let $G$ be a discrete QCF group acting on $\overline{\mathbb{B}^{n}}$. Then $\delta(G) \leq n-1$.

We note that for Kleinian groups, Sullivan [22], and separately Tukia [24], provided an important refinement to the above by showing that a geometrically finite Kleinian group acting on $\overline{\mathbb{B}^{n}}$ and with nonempty regular set has an exponent of convergence that is strictly less than $n-1$. We have recently extended this result to QCF groups having purely conical limit sets (see [1]).

For a non-elementary QCF group $G$, we can refine the trivial lower bound.

Theorem 3.3. Let $G$ be a non-elementary discrete $Q C F$ group acting on $\overline{\mathbb{B}^{n}}$. Then $\delta(G)>0$.

To show this lower bound, we need the next two results.

Theorem 3.4 (Theorem 3.3 in [5]). Let $G$ and $\tilde{G}$ be two discrete QCF groups acting on $\overline{\mathbb{B}^{n}}$ which agree on $\mathbb{S}^{n-1}$, i.e., there exists an isomorphism $\varphi: G \rightarrow \tilde{G}$ such that $g_{\mid \mathbb{S}^{n-1}}=\varphi(g)_{\mid \mathbb{S}^{n-1}}$ for all $g \in G$. Then $\delta(G)=\delta(\tilde{G})$.

The following result of G. Martin 13 shows that, even if a discrete QCF group is not itself conjugate to any Kleinian group, we can find a non-elementary subgroup that is.

Lemma 3.5. For any non-elementary quasiconformal group $G$ acting on $\overline{\mathbb{R}^{n}}(n \in$ $\mathbb{N}, n \neq 4$ ) there exists a non-elementary subgroup $H$ that is quasiconformally (quasisymmetrically when $n=1$ ) conjugate to a Kleinian group $\Gamma$.

Proof of Lemma 3.5. When $n \leq 2$ the entire group $G$ is quasiconformally (quasisymmetrically) conjugate to a Kleinian group [21, 25]. Thus we may now assume that $n \geq 3$.

Because $G$ is non-elementary, there exists a free rank 2, purely loxodromic subgroup $H$ of $G$ that has nonempty regular set. Thus $L(H)$ is a Cantor set, $\Omega(H)$ is simply connected $(n \geq 3)$, and $\Omega(H) / H$ is compact. Via a result of FreedmanSkora 7] we observe that $\Omega(H) / H$ is topologically equivalent to a connected sum of two copies of $\mathbb{S}^{n-1} \times \mathbb{S}^{1}$.

Note that $\Omega(H) / H$ inherits a quasiconformal topological structure from the action of $H \subset G$. Sullivan [19] shows that this structure is unique up to quasiconformal conjugacy $(n \neq 4)$. It is easy to see that for any 2-generator classical (Kleinian) Schottky group $\Gamma$, the quotient $\Omega(\Gamma) / \Gamma$ is also homeomorphic to a connected sum of two copies of $\mathbb{S}^{n-1} \times \mathbb{S}^{1}$, and thus by the above-mentioned result of Sullivan, there exists a quasiconformal mapping $f: \Omega(H) \rightarrow \Omega(\Gamma)$ that conjugates $H$ to $\Gamma$. 
The mapping $f$ extends to a quasiconformal mapping $f: \overline{\mathbb{R}^{n}} \rightarrow \overline{\mathbb{R}^{n}}$ preserving the conjugacy.

Remark 3.6. The argument in the proof of Lemma 3.5 works for any finite choice of rank.

We now finish the proof of Theorem 3.3.

Proof of Theorem 3.3. First, let $n \neq 5$. Because $G$ is a QCF group acting on $\overline{\mathbb{B}^{n}}$, the sphere $\mathbb{S}^{n-1} \cong \overline{\mathbb{R}}^{n-1}$ is invariant under $G$, and we restrict our attention to this sphere for now. Note that since $n \neq 5$, the dimension of the sphere is not equal to 4 , and we can thus apply Lemma3.5. Hence there exists a non-elementary subgroup $H$ of $G$ acting on $\mathbb{S}^{n-1}$ that is quasiconformally conjugate (via a map $\left.\varphi: \mathbb{S}^{n-1} \rightarrow \mathbb{S}^{n-1}\right)$ to a Kleinian group $\Gamma$ on $\mathbb{S}^{n-1}$. The Kleinian group is a nonelementary Schottky group, and therefore $\delta(\Gamma)>0$. Extending $\Gamma$ and $\varphi$ to $\mathbb{B}^{n}$ we define a QCF group $\widetilde{H}:=\varphi \Gamma \varphi^{-1}$ on $\overline{\mathbb{B}^{n}}$ that agrees with $H$ on $\mathbb{S}^{n-1}$. Thus $\delta(H)=\delta(\widetilde{H})$ (Theorem [3.4), and using the Hölder continuity of $\varphi$, we observe that $\delta(\widetilde{H})>0$.

Now let $n=5$. Extend the group $G$ via reflection to a quasiconformal group acting on $\overline{\mathbb{R}^{n}}$. Using Lemma 3.5 we can find a non-elementary subgroup $H$ of $G$ acting on $\overline{\mathbb{R}^{n}}$ that is quasiconformally conjugate to a Kleinian group $\Gamma$ on $\overline{\mathbb{R}^{n}}$. Since both $\Gamma$ and the conjugating map extend to $\mathbb{H}^{n+1}$, we can also extend $H$ to a quasiconformal group $\widetilde{H}$ acting on $\mathbb{H}^{n+1}$. It is easy to see that $\delta(\widetilde{H})$ (computed in $\mathbb{H}^{n+1}$ ) equals $\delta(H)$ (computed in $\left.\mathbb{B}^{n}\right)$. Since $\Gamma$ is a non-elementary Schottky group, we have $\delta(\Gamma)>0$, and again using Hölder continuity of the conjugating map we obtain $\delta(\widetilde{H})>0$ and so $\delta(H)>0$.

Our purpose in this paper is to explore the relationship between the exponent of convergence and the Hausdorff dimension of the limit set of a QCF group. In the setting of Kleinian groups, there is a deep connection between Hausdorff dimension and the exponent of convergence.

Theorem 3.7. Let $\Gamma$ be a non-elementary Kleinian group.

(1) If $\Gamma$ is geometrically finite, then $\delta(\Gamma)=\operatorname{dim}(L(\Gamma))$ [17], [20].

(2) Without additional assumptions, we have that $\delta(\Gamma)=\operatorname{dim}\left(L_{c}(\Gamma)\right)[3$.

As we will show, the theory is not so concise in the setting of QCF groups. As our point of departure we note the following general result (see Theorem 2.7 in [5]):

Theorem 3.8. Let $G$ be a discrete QCF group. Then $\operatorname{dim}\left(L_{c}(G)\right) \leq \delta(G)$.

In particular, there are examples of QCF groups $G$ that arise as quasiconformal conjugates of geometrically finite Kleinian groups, so that $\operatorname{dim}\left(L_{c}(G)\right)<\delta(G)$ (see Example 8.1).

\section{The Construction of a Patterson-Sullivan Measure}

Let $G$ be a discrete QCF group acting on $\overline{\mathbb{B}^{n}}$. We show that the PattersonSullivan construction [20], [16] yields a measure $\mu_{x, y}^{\delta(G)}\left(x, y \in \mathbb{B}^{n}\right)$ supported on the limit set of $G$. The following properties of Patterson-Sullivan measures remain true as in the conformal case:

- The measure is wholly supported on (possibly a proper subset of) the limit set $L(G)$ of $G$; it is finite and positive there. 
- For any other choice of $x^{\prime} \in \mathbb{B}^{n}$, the measures $\mu_{x, y}^{\delta(G)}$ and $\mu_{x^{\prime}, y}^{\delta(G)}$ are absolutely continuous with respect to each other.

Recall that for a Kleinian group $\Gamma$, the Patterson-Sullivan construction results in a $\Gamma$-invariant function, called a $\Gamma$-invariant conformal density, from a space of Riemannian metrics on $\mathbb{S}^{n-1}$ to a space of positive finite measures on $\mathbb{S}^{n-1}$. As alluded to above, each resulting Patterson-Sullivan measure $\mu_{x, y}^{\delta(\Gamma)}$ satisfies the above two properties, as well as the following transformation rule for all $\gamma \in \Gamma$ :

$$
\gamma * \mu_{x, y}^{\delta(\Gamma)}=\mu_{\gamma^{-1}(x), y}^{\delta(\Gamma)}
$$

where $\gamma * \mu_{x, y}^{\delta(\Gamma)}(A)=\mu_{x, y}^{\delta(\Gamma)}(\gamma A)$.

Crucially, for a generic QCF group $G$, the measures $\mu_{x, y}^{\delta(G)}$ do not satisfy this transformation property: For $g \in G, g * \mu_{x, y}^{\delta(G)}(A) \neq \mu_{g^{-1}(x), y}^{\delta(G)}(A)$ for "most sets" $A$. This is to say that the Patterson-Sullivan construction does not build a $G$-invariant conformal density on most discrete quasiconformal groups.

The construction of the Patterson-Sullivan measure for QCF groups is in all essential respects the same as the construction for Kleinian groups. We will closely follow the proof given by D. Sullivan in Section 1 of [20, with certain analytic details provided in more detail.

Fix a discrete QCF group $G$ acting on $\overline{\mathbb{B}^{n}}$. For ease of exposition, let $\delta=\delta(G)$. Following Sullivan [20], let $s>\delta$ and fix $x, y \in \mathbb{B}^{n}$; define

$$
\mu_{x, y}^{s}=\frac{1}{\Sigma(s, y, y)} \sum_{g \in G} e^{-s \rho(x, g(y))} \delta(g(y)),
$$

where $\delta(g(y))$ is the Dirac measure supported on the orbit point $g(y) \in \mathbb{B}^{n}$. Since $\Sigma(s, y, y)<\infty$, it is easy to see that the mass of $\mu_{x, y}^{s}$ is positive and finite, and that the support of this measure is the closure of the collection of orbit points $G(y)=\left\{\bigcup_{g \in G} g(y)\right\}$.

Fix a sequence of exponents $\left\{s_{i}: s_{i}>\delta\right\}$ that is converging (from above) to $\delta$, where we may assume, without loss of generality, that $s_{i} \leq 2 \delta$ for all $i$. For now we assume that $\Sigma(\delta, y, y)=\infty$; we will construct the measure under this assumption and then show how to handle the case that $\Sigma(\delta, y, y)<\infty$.

By the triangle inequality, we have that

$$
e^{-s \rho(x, y)} \Sigma(s, y, y) \leq \Sigma(s, x, y) \leq e^{s \rho(x, y)} \Sigma(s, y, y),
$$

and so for each index $i$ we have $e^{-2 \delta \rho(x, y)}<\operatorname{mass} \mu_{x, y}^{s_{i}}<e^{2 \delta \rho(x, y)}$. Thus for each $i$, we can find a positive real number $m_{i} \in\left(e^{-2 \delta \rho(x, y)}, e^{2 \delta \rho(x, y)}\right)$ so that $\hat{\mu}_{x, y}^{s_{i}}=\frac{1}{m_{i}} \mu_{x, y}^{s_{i}}$ is in the space $M_{1}\left(\overline{\mathbb{B}^{n}}\right)$ of probability measures on $\overline{\mathbb{B}^{n}}$. Let $M=$ $\left\{\hat{\mu}_{x, y}^{s_{1}}, \hat{\mu}_{x, y}^{s_{2}}, \ldots, \hat{\mu}_{x, y}^{s_{n}}, \ldots\right\}$, and let $\bar{M}$ be the closure of $M$ in the weak topology on $M_{1}\left(\overline{\mathbb{B}^{n}}\right)$. (For the basics on topologies on spaces of probability measures, see Chapter 3 of [18].) Using Theorem 3.1.9 in 18 we can show that $\bar{M}$ is compact in the weak topology.

Hence we can find a measure, denoted by $\hat{\mu}_{x, y}^{\delta}$, and a subsequence $\left\{\hat{\mu}_{x, y}^{s_{i_{k}}}\right\} \in M$ so that $\hat{\mu}_{x, y}^{s_{i}} \rightarrow \hat{\mu}_{x, y}^{\delta}$ in the weak topology. Since the $m_{i}$ are uniformly bounded above and below, we can find a subsequence of the subsequence, so that $m_{i_{k}} \rightarrow m_{\infty}$. Thus we observe that $\left\{\mu_{x, y}^{s_{i}}\right\}$ converges to $\mu_{x, y}^{\delta}=m_{\infty} \cdot \hat{\mu}_{x, y}^{\delta}$.

Because of the assumption that $\Sigma(\delta, y, y)=\infty$, it is clear that the support of $\mu_{x, y}^{s_{i}}$ is swept off of $G(y)$ in the limit as $s_{i} \rightarrow \delta(G)^{+}$, and it is not hard to show that the 
support of $\mu_{x, y}^{\delta}$ is in the limit set $L(G)$ ([16], Theorem 3.3.2). It is a consequence of weak convergence that $\mu_{x, y}^{\delta}$ is finite and positive on $L(G)$ : the Euclidean closure of the orbit $G(y)$ is closed, and so we obtain a positive lower bound on the mass of $\mu_{x, y}^{\delta}$ from the uniform lower bounds on the masses of $\left\{\mu_{x, y}^{s_{k}}\right\}$ (see [18], Theorem 3.1.5(iii)). For the upper bound, consider (in the Euclidean topology) a covering of the limit set by open hemispheres in $\overline{\mathbb{B}^{n}}$. Here we recover an upper bound from the uniform upper bounds on the masses of $\left\{\mu_{x, y}^{s_{k}}\right\}$. (See [18], Theorem 3.1.5(iv).)

We observe that if the series $\Sigma(\delta, y, y)$ converges, then the limit measure puts mass on $G(y)$; thus we need to "scale" the Poincaré series so that the resulting limit measure has its support solely on the limit set. This scaling is accomplished using an argument of Patterson given in [17. Since $G$ acts discontinuously on $\mathbb{B}^{n}$ we can order all $g_{j} \in G$ so that $\rho\left(x, g_{j}(y)\right) \nearrow \infty$ as $j \rightarrow \infty$. One can construct a scaling function $h: \mathbb{R}^{+} \rightarrow \mathbb{R}^{+}$(see Lemma 3.1.1 in [16] for the details) so that:

(1) The function $h$ is piecewise continuous and increasing.

(2) The scaled Poincaré series

$$
\Sigma^{*}(s, y, y):=\sum_{g \in G} h\left(e^{\rho(x, g(y))}\right) e^{-s \rho(x, g(y))}
$$

converges for $s<\delta$ and diverges for $s \geq \delta$.

(3) For a fixed $\varepsilon>0$, the function $h$ satisfies the order relation

$$
h\left(e^{\rho\left(x, g_{j}(y)\right)}\right)=O\left(\left(e^{\rho\left(x, g_{j}(y)\right)}\right)^{\varepsilon}\right) .
$$

One now defines analogously a new measure on $G(y)$ for $s>\delta$,

$$
\mu_{x, y}^{s}=\frac{1}{\Sigma^{*}(s, y, y)} \sum_{g \in G} e^{-s \rho(x, g(y))} h\left(e^{\rho(x, g(y))}\right) \delta(g(y))
$$

and follows the procedure given above.

A question remains as to how the Patterson-Sullivan construction changes under a different choice of basepoint. So we now establish the relationship between $\mu_{x, y}^{\delta}$ and $\mu_{x^{\prime}, y}^{\delta}$, where $x \neq x^{\prime}$. For $(x, \zeta) \in \mathbb{B}^{n} \times \mathbb{S}^{n-1}$, recall that the Poisson kernel is given by $P(x, \zeta)=\frac{1-|x|^{2}}{|x-\zeta|^{2}}$.

Lemma 4.1. Fix two pairs of points $(x, y),\left(x^{\prime}, y\right) \in \mathbb{B}^{n} \times \mathbb{B}^{n}$, where $x \neq x^{\prime}$. Then the Radon-Nikodym derivative of $\mu_{x^{\prime}, y}^{\delta}$ with respect to $\mu_{x, y}^{\delta}$ satisfies

$$
\left(\frac{d \mu_{x^{\prime}, y}^{\delta}}{d \mu_{x, y}^{\delta}}\right)(\zeta)=\left(\frac{P\left(x^{\prime}, \zeta\right)}{P(x, \zeta)}\right)^{\delta}
$$

for $\zeta \in \mathbb{S}^{n-1}$.

Proof of Lemma 4.1. We provide an outline of the proof encapsulating the essential geometric ideas.

Since the Patterson-Sullivan measure is supported on a set in the limit set, we need only consider a fixed $\zeta \in L(G)$. We wish to compare $\mu_{x^{\prime}, y}^{s}$ with $\mu_{x, y}^{s}$ as $s \rightarrow \delta$, and thus we need an asymptotic expression for $e^{-s\left(\rho\left(x^{\prime}, g(y)\right)-\rho(x, g(y))\right)}$, where $g \in G$ so that $g(y)$ is arbitrarily close in the chordal metric to $\zeta$. Using an identity from hyperbolic trigonometry involving the hyperbolic sine (equation 7.2.4 in [2]), we 
observe that $e^{\rho\left(x^{\prime}, g(y)\right)}$ is asymptotically $\frac{1}{P\left(x^{\prime}, \zeta\right)}$, and likewise for $e^{\rho(x, g(y))}$. Thus we have

$$
e^{-s\left(\rho\left(x^{\prime}, g(y)\right)-\rho(x, g(y))\right)} \sim\left(\frac{P\left(x^{\prime}, \zeta\right)}{P(x, \zeta)}\right)^{s} .
$$

Fix the two horospheres $H^{\prime}$ and $H$ based at $\zeta$ and through $x^{\prime}, x$ respectively. Then $\frac{P\left(x^{\prime}, \zeta\right)}{P(x, \zeta)}$ admits a nice geometric interpretation: it is the exponential of the signed hyperbolic distance between $H^{\prime}$ and $H$.

Because $\Sigma^{*}(s, y, y) \rightarrow \infty$ as $s \rightarrow \delta^{+}$, we see that only the orbit points "near" $\zeta$ need be considered.

To finish the proof of the lemma it only needs to be shown, for any sufficiently small open neighborhood of $\zeta$ in the (Euclidean) subspace topology, that the ratio of the measures $\mu_{x^{\prime}, y}^{\delta}$ to $\mu_{x, y}^{\delta}$ is bounded (from above and below) in terms of $\left(\frac{P\left(x^{\prime}, \zeta\right)}{P(x, \zeta)}\right)^{\delta}$. (See Theorem 3.4.1 in [16].)

Remark 4.2. As in the case of the scaling function $h$, Lemma 4.1 depends only on the discontinuous action of $G$ on $\mathbb{B}^{n}$. It is the isometric action of a Kleinian group $\Gamma$ on $\left(\mathbb{B}^{n}, \rho\right)$ that allows one to interpret $\left(\frac{P\left(x^{\prime}, \zeta\right)}{P(x, \zeta)}\right)^{\delta}$ geometrically; it is from this geometric interpretation that the transformation property (as given in equation (4.1)) of Patterson-Sullivan measures is deduced. In Section 7 we will see that typically, in the quasiconformal case, the Patterson-Sullivan measures are supported on a proper subset of the limit set, and this implies that one cannot expect the transformation property to hold in general. Moreover, in Section 5 we shall see that, under some additional assumptions, the transformation property implies that the QCF group is Kleinian.

We collect the results of the construction above in the following statement:

Theorem 4.3 (Patterson, Sullivan). Let $G$ be a discrete $Q C F$ group acting on $\overline{\mathbb{B}^{n}}$, and fix $(x, y) \in \mathbb{B}^{n} \times \mathbb{B}^{n}$. Let $\delta=\delta(G)$ be the exponent of convergence for the Poincaré series of $G$. Then:

(1) For a choice of $\left\{s_{i}: s_{i} \rightarrow \delta^{+}\right\}$there exists a Patterson-Sullivan measure $\mu_{x, y}^{\delta}$ which is the weak limit of a subsequence of $\left\{\mu_{x, y}^{s_{i}}\right\}$. The measure $\mu_{x, y}^{\delta}$ is wholly supported on the limit set $L(G)$, and is finite and positive there.

(2) If $x^{\prime} \neq x$, then $\mu_{x, y}^{\delta}$ is absolutely continuous with respect to $\mu_{x^{\prime}, y}^{\delta}$, with the Radon-Nikodym derivative being given as in Lemma 4.1 .

\section{The Transformation Property}

We prove a measure-theoretic regularity result in this section: we show that a non-elementary discrete QCF group acting on $\overline{\mathbb{B}^{n}}$ whose associated PattersonSullivan measures satisfy the transformation property is in fact a Kleinian group.

Theorem 5.1. Let $G$ be a non-elementary discrete $Q C F$ group acting on $\overline{\mathbb{B}^{n}}$ and suppose that the Patterson-Sullivan measures associated to $G$ satisfy the transformation property, i.e., there exists $y \in \mathbb{B}^{n}$ so that

$$
\mu_{g^{-1}(x), y}^{\delta}=g * \mu_{x, y}^{\delta} \quad \text { for all } x \in \mathbb{B}^{n} \text { and all } g \in G .
$$

Then $G$ is a Kleinian group. 
Proof of Theorem [5.1. Fix $y \in \mathbb{B}^{n}$ such that (5.1) holds for all $x \in \mathbb{B}^{n}$ and all $g \in G$. Using Lemma 4.1 we then have for a measurable set $E \subset L(G)$ and $g \in G$ that

$$
\begin{aligned}
\int_{g(E)} \mu_{0, y}^{\delta}(d \zeta) & =g * \mu_{0, y}^{\delta}(E) \\
& =\mu_{g^{-1}(0), y}^{\delta}(E) \\
& =\int_{E}\left(\frac{P\left(g^{-1}(0), \zeta\right)}{P(0, \zeta)}\right)^{\delta} \mu_{0, y}^{\delta}(d \zeta)
\end{aligned}
$$

By looking at limits of simple functions this implies that for a measurable function $\varphi$ on $L(G)$ and $g \in G$ we have the following transformation rule:

$$
\int_{g(E)} \varphi(\zeta) \mu_{0, y}^{\delta}(d \zeta)=\int_{E} \varphi(g(\zeta))\left(\frac{P\left(g^{-1}(0), \zeta\right)}{P(0, \zeta)}\right)^{\delta} \mu_{0, y}^{\delta}(d \zeta)
$$

Now let $x \in \mathbb{B}^{n}$ and $g \in G$. Then, using (5.2) we obtain that

$$
\begin{aligned}
\mu_{x, y}^{\delta}(g(E)) & =\int_{g(E)}\left(\frac{P(x, \zeta)}{P(0, \zeta)}\right)^{\delta} \mu_{0, y}^{\delta}(d \zeta) \\
& =\int_{E}\left(\frac{P(x, g(\zeta))}{P(0, g(\zeta))}\right)^{\delta}\left(\frac{P\left(g^{-1}(0), \zeta\right)}{P(0, \zeta)}\right)^{\delta} \mu_{0, y}^{\delta}(d \zeta) .
\end{aligned}
$$

On the other hand, using (5.1) and Lemma 4.1 we have that

$$
\begin{aligned}
\mu_{x, y}^{\delta}(g(E)) & =\mu_{g^{-1}(x), y}^{\delta}(E) \\
& =\int_{E}\left(\frac{P\left(g^{-1}(x), \zeta\right)}{P(0, \zeta)}\right)^{\delta} \mu_{0, y}^{\delta}(d \zeta) .
\end{aligned}
$$

Hence we see that

$$
\int_{E}\left(\frac{P(x, g(\zeta))}{P(0, g(\zeta))}\right)^{\delta}\left(\frac{P\left(g^{-1}(0), \zeta\right)}{P(0, \zeta)}\right)^{\delta} \mu_{0, y}^{\delta}(d \zeta)=\int_{E}\left(\frac{P\left(g^{-1}(x), \zeta\right)}{P(0, \zeta)}\right)^{\delta} \mu_{0, y}^{\delta}(d \zeta)
$$

for all $x \in \mathbb{B}^{n}, g \in G$, and measurable $E \subset L(G)$.

Since $E$ was an arbitrary measurable subset of $L(G)$ we conclude that, for fixed $x \in \mathbb{B}^{n}$ and fixed $g \in G$, we have

$$
\left(\frac{1-|x|^{2}}{|x-g(\zeta)|^{2}}\right)^{\delta}\left(\frac{1-\left|g^{-1}(0)\right|^{2}}{\left|g^{-1}(0)-\zeta\right|^{2}}\right)^{\delta}=\left(\frac{1-\left|g^{-1}(x)\right|^{2}}{\left|g^{-1}(x)-\zeta\right|^{2}}\right)^{\delta}
$$

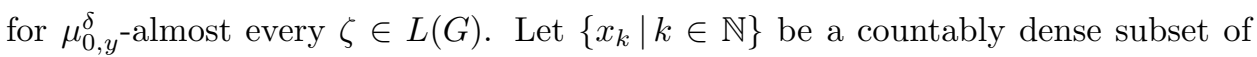
$\mathbb{B}^{n}$. Then there exists a set $N \subset L(G)$ with $\mu_{0, y}^{\delta}(N)=0$ such that

$$
\left(\frac{1-\left|x_{k}\right|^{2}}{\left|x_{k}-g(\zeta)\right|^{2}}\right)^{\delta}\left(\frac{1-\left|g^{-1}(0)\right|^{2}}{\left|g^{-1}(0)-\zeta\right|^{2}}\right)^{\delta}=\left(\frac{1-\left|g^{-1}\left(x_{k}\right)\right|^{2}}{\left|g^{-1}\left(x_{k}\right)-\zeta\right|^{2}}\right)^{\delta}
$$

holds for all $k \in \mathbb{N}$ and all $\zeta \in L(G) \backslash N$. Since $\mu_{0, y}^{\delta}(L(G))>0$ by Theorem 4.3 , the set $F:=L(G) \backslash N$ has positive $\mu_{0, y}^{\delta}$-measure. Then (5.3) implies that for $\zeta \in F$, the quantity

$$
\frac{1-\left|g^{-1}\left(x_{k}\right)\right|^{2}}{\left|g^{-1}\left(x_{k}\right)-\zeta\right|^{2}} \frac{\left|x_{k}-g(\zeta)\right|^{2}}{1-\left|x_{k}\right|^{2}}
$$


is independent of $k$, and thus by continuity of $g$ and the density of $\left\{x_{k} \mid k \in \mathbb{N}\right\}$ in $\mathbb{B}^{n}$ we conclude that

$$
\frac{1-\left|g^{-1}(x)\right|^{2}}{\left|g^{-1}(x)-\zeta\right|^{2}} \frac{|x-g(\zeta)|^{2}}{1-|x|^{2}}
$$

is constant in $x \in \mathbb{B}^{n}$ for each (fixed) $\zeta \in F$.

From the facts that $\mu_{0, y}^{\delta}(L(G))>0, \mu_{0, y}^{\delta}(L(G) \backslash F)=0$, and (5.1) it follows that $F$ has infinitely many points. The theorem now follows by applying the lemma below.

Lemma 5.2. Let $g: \overline{\mathbb{B}^{n}} \rightarrow \overline{\mathbb{B}^{n}}$ be an orientation-preserving homeomorphism so that the quantity

$$
\left(\frac{|g(y)-g(\zeta)|^{2}}{1-|g(y)|^{2}}\right) \cdot\left(\frac{1-|y|^{2}}{|y-\zeta|^{2}}\right)
$$

is independent of $y \in \mathbb{B}^{n}$ for each (fixed) $\zeta \in L \subset \mathbb{S}^{n-1}$, where $L$ has at least $n+1$ points. Then $g$ is a Möbius transformation.

Proof of Lemma 5.2. We will give the proof in the case $n=2$ for ease of visualization. The general case is an easy modification.

Let $g: \overline{\mathbb{B}^{2}} \rightarrow \overline{\mathbb{B}^{2}}$ be as in the statement of the lemma, and let $\zeta_{0} \in L$. Choose a Möbius transformation $\gamma$ preserving the unit ball such that $\gamma(g(0))=0$, and $\gamma\left(g\left(\zeta_{0}\right)\right)=\zeta_{0}$. One can easily show that $f:=\gamma \circ g$ satisfies the assumptions of the lemma, and by construction fixes 0 and $\zeta_{0}$. We will show that this implies that $f=$ id, and hence $g=\gamma^{-1}$.

We have that the quantity

$$
\frac{|f(y)-f(\zeta)|^{2}}{1-|f(y)|^{2}} \frac{1-|y|^{2}}{|y-\zeta|^{2}}
$$

is independent of $y \in \mathbb{B}^{2}$ for each (fixed) $\zeta \in L$, where $L$ has at least 3 elements. Since $f(0)=0$ this quantity equals 1 for each $y \in \mathbb{B}^{2}$ and each $\zeta \in L$. This implies that the image under $f$ of a horoball of radius $r<1$ based at $\zeta \in L$ is a horoball based at $f(\zeta)$ of the same radius $r$. In particular, any horoball based at $\zeta_{0}$ is (setwise) fixed under $f$.

Now let $\zeta \in L, \zeta \neq \zeta_{0}$. We can conclude that any horoball based at $\zeta$ is setwise preserved under $f$ as well: Let $r$ be the radius of a horoball at $\zeta$. Then there is a unique horoball of some radius $\tilde{r}$ based at $\zeta_{0}$ that touches the horoball of radius $r$ based at $\zeta$, and since the former ball is fixed under $f$ and the latter horoball maps onto a horoball of radius $r$, using the fact that $f$ is an orientation-preserving homeomorphism we obtain that $f$ fixes the horoball of radius $r$ at $\zeta$ setwise, and so $f(\zeta)=\zeta$.

Thus we conclude that $f$ fixes each $\zeta \in L$.

Now let $z \in \mathbb{B}^{2}$ be arbitrary. Let $\zeta_{1}, \zeta_{2}, \zeta_{3} \in L$ be three distinct points. Then the horoballs based at $\zeta_{1}, \zeta_{2}$ and $\zeta_{3}$ respectively, that contain $z$, intersect at exactly one point: $z$. Since all these horoballs are setwise preserved under $f$, the map $f$ must fix $z$.

\section{The Local Analysis of the Limit Set}

In [5] we show that any discrete QCF group acting on $\overline{\mathbb{B}^{n}}$ satisfies $\delta(G) \leq$ $\operatorname{dim} L_{c}(G)$. We furthermore provide an example of a discrete quasiconformal group 
$G$ acting on $\mathbb{H}^{2}$ that satisfies $\operatorname{dim} L_{c}(G)<\delta(G)$ and that is quasiconformally conjugate to a finitely generated Fuchsian group of the second kind containing a parabolic element fixing the point $\infty$. In this example, the conjugating map is locally bi-Lipschitz at every $x \in \mathbb{R}$ but not at $\infty$. Thus, the phenomenon that produces the strict inequality between the exponent of convergence and the Hausdorff dimension of the conical limit set is localized around the parabolic fixed point $\infty$. This observation prompts us to develop localized versions of the exponent of convergence and of the Hausdorff dimension.

In this section we define the local exponent of convergence and the local dimension of the limit set, and we prove some basic properties. In the next section we will relate these results to Patterson-Sullivan measures associated with a discrete QCF group and show that their support is contained in the set of limit points where the local exponent is maximal.

We will study the example mentioned above with respect to these local quantities in Section 8.

Definition 6.1. Let $G$ be a discrete QCF group acting on $\overline{\mathbb{B}^{n}}$. For $x \in \mathbb{S}^{n-1}$ and $r>0$ define

$$
\delta_{x}^{r}(G):=\inf \left\{s>0 \mid \sum_{g \in G: g(0) \in B_{r}^{n}(x)} e^{-s \rho(0, g(0))}<\infty\right\},
$$

where $B_{r}^{n}(x)$ is the Euclidean $n$-dimensional ball of radius $r$ around $x \in \mathbb{S}^{n-1} \subset \mathbb{R}^{n}$. Furthermore, define the local exponent of convergence of $G$ at $x$ to be

$$
\delta_{x}(G):=\lim _{r \rightarrow 0} \delta_{x}^{r}(G) .
$$

Remark 6.2. Note that for $r_{1}<r_{2}$ we have $B_{r_{1}}^{n}(x) \subset B_{r_{2}}^{n}(x)$, and thus $\delta_{x}^{r_{1}}(G) \leq$ $\delta_{x}^{r_{2}}(G)$. Clearly $0 \leq \delta_{x}(G) \leq \delta(G)$, and hence the above limit exists.

We begin our investigation by showing some elementary properties of the local exponent of convergence. For $x \in \mathbb{S}^{n-1}$ and $r>0$ let

$$
\dot{B}_{r}^{n-1}(x):=\left\{w \in \mathbb{S}^{n-1}|0<| w-x \mid<r\right\} .
$$

Lemma 6.3. The local exponent of convergence satisfies the following properties:

(1) $\delta_{x}(G)=0$ for $x \notin L(G)$.

(2) $\delta(G)=\max _{x \in L(G)} \delta_{x}(G)$.

(3) $\delta_{x}(G) \geq \lim _{r \rightarrow 0} \sup \left\{\delta_{w}(G) \mid w \in \dot{B}_{r}^{n-1}(x)\right\}$ for all $x \in \mathbb{S}^{n-1}$.

We show (Example 8.1) that ">" can occur in 6.3 (3).

Complimentary to studying local convergence properties of QCF groups we also study local properties of limit sets of QCF groups.

Definition 6.4. Let $G$ be a discrete QCF group acting on $\overline{\mathbb{B}^{n}}$. For $x \in \mathbb{S}^{n-1} \subset \mathbb{R}^{n}$ define

$$
\operatorname{dim}_{x}(L(G)):=\lim _{r \rightarrow 0} \operatorname{dim}\left(B_{r}^{n}(x) \cap L(G)\right),
$$

where dim denotes the Hausdorff dimension. We call $\operatorname{dim}_{x}(G)$ the local dimension of $L(G)$ at $x$.

As with the local exponent of convergence we will show some elementary properties of the local dimension of $L(G)$.

Lemma 6.5. The local dimension of $L(G)$ satisfies the following properties: 
(1) $\operatorname{dim}_{x}(L(G))=0$ for $x \notin L(G)$;

(2) $\operatorname{dim}(L(G))=\max _{x \in L(G)} \operatorname{dim}_{x}(L(G))$;

(3) $\operatorname{dim}_{x}(L(G))=\lim _{r \rightarrow 0} \sup \left\{\operatorname{dim}_{w}(L(G)) \mid w \in \dot{B}_{r}^{n-1}(x)\right\}$ for all $x \in \mathbb{S}^{n-1}$.

Note that in (3) of Lemma 6.5 we have equality, whereas in the corresponding property (3) in Lemma 6.3 we only have " $\leq$ ".

Likewise we define the local dimension of the conical limit set of $G$ at $x$ to be

$$
\operatorname{dim}_{x}\left(L_{c}(G)\right):=\lim _{r \rightarrow 0} \operatorname{dim}\left(B_{r}(x) \cap L_{c}(G)\right) .
$$

Lemma 6.5 then becomes:

Lemma 6.6. The local dimension of $L_{c}(G)$ satisfies the following properties:

(1) $\operatorname{dim}_{x}\left(L_{c}(G)\right)=0$ for $x \notin \overline{L_{c}(G)}=L(G)$;

(2) $\operatorname{dim}\left(L_{c}(G)\right)=\sup _{x \in L_{c}(G)} \operatorname{dim}_{x}\left(L_{c}(G)\right)=\max _{x \in L(G)} \operatorname{dim}_{x}\left(L_{c}(G)\right)$;

(3) $\operatorname{dim}_{x}\left(L_{c}(G)\right)=\lim _{r \rightarrow 0} \sup \left\{\operatorname{dim}_{w}\left(L_{c}(G)\right) \mid w \in \dot{B}_{r}^{n-1}(x)\right\}$ $=\lim _{r \rightarrow 0} \sup \left\{\operatorname{dim}_{w}\left(L_{c}(G)\right) \mid w \in \dot{B}_{r}^{n-1}(x) \cap L_{c}(G)\right\}$ for all $x \in \mathbb{S}^{n-1}$.

We can relate the local exponent of convergence with the local dimension of the conical limit set:

Theorem 6.7. Let $G$ be a discrete $Q C F$ group acting on $\overline{\mathbb{B}^{n}}$. Then for every $x \in \mathbb{S}^{n-1}$ we have that $\operatorname{dim}_{x}\left(L_{c}(G)\right) \leq \delta_{x}(G)$.

Combining Theorem [6.7, Lemma 6.3 and Lemma 6.6] we establish a local proof of Theorem 2.7 in [5]:

Theorem 6.8. Let $G$ be a discrete $Q C F$ group acting on $\overline{\mathbb{B}^{n}}$. Then $\operatorname{dim}\left(L_{c}(G)\right) \leq$ $\delta(G)$.

We will see (Example 8.1) that " $<$ " can occur in this theorem.

In the setting of discrete Möbius groups, considering the local exponent of convergence and the local Hausdorff dimension results in a basic fact from PattersonSullivan theory.

Theorem 6.9. Let $\Gamma$ be a Fuchsian group acting on $\overline{\mathbb{B}^{n}}$, i.e., $\Gamma$ is a discrete group of Möbius transformation each of which keeps $\mathbb{B}^{n}$ invariant. Then

(1) $\delta_{x}(\Gamma)=\delta(\Gamma)$ for all $x \in L(\Gamma)$;

(2) $\operatorname{dim}_{x}(L(\Gamma))=\operatorname{dim}(L(\Gamma))$ for all $x \in L(\Gamma)$;

(3) $\operatorname{dim}_{x}\left(L_{c}(\Gamma)\right)=\operatorname{dim}\left(L_{c}(\Gamma)\right)$ for all $x \in L(\Gamma)$;

(4) if $\Gamma$ is non-elementary, then $\operatorname{dim}_{x}\left(L_{c}(\Gamma)\right)=\delta_{x}(\Gamma)$ for all $x \in L(\Gamma)$.

Although we use arguments involving the hyperbolic metric, of course QCF groups (non-Kleinian) do not act isometrically on $\mathbb{B}^{n}$. However, each element and its inverse are almost everywhere differentiable on $\mathbb{S}^{n-1}$, and this implies that each element is almost everywhere a local bi-Lipschitz mapping (with respect to the Euclidean metric). The usefulness of the local exponent of convergence and the local Hausdorff dimension are that they are bi-Lipschitz invariants.

Theorem 6.10. Let $G$ be a discrete $Q C F$ group acting on $\overline{\mathbb{B}^{n}}$, and let $\varphi: \overline{\mathbb{B}^{n}} \rightarrow \overline{\mathbb{B}^{n}}$ be a quasiconformal homeomorphism preserving $\mathbb{B}^{n}$. Define $\widetilde{G}:=\varphi \circ G \circ \varphi^{-1}$. Suppose there exists $w \in \mathbb{S}^{n-1}$ and $r>0, L>0$ such that

$$
\frac{1}{L}|x-y| \leq|\varphi(x)-\varphi(y)| \leq L|x-y|
$$

for all $x, y \in B_{r}(w) \cap \mathbb{S}^{n-1}$. Then: 
(1) $\delta_{\varphi(w)}(\widetilde{G})=\delta_{w}(G)$;

(2) $\operatorname{dim}_{\varphi(w)}(L(\widetilde{G}))=\operatorname{dim}_{w}(L(G))$;

(3) $\operatorname{dim}_{\varphi(w)}\left(L_{c}(\widetilde{G})\right)=\operatorname{dim}_{w}\left(L_{c}(G)\right)$.

We now prove the above lemmas and theorems.

Proof of Lemma 6.3 $\quad$ (1) If $x \in \mathbb{S}^{n-1} \backslash L(G)$, then there exists $R>0$ such that only finitely many points of the orbit of 0 under $G$ lie in $B_{R}^{n}(x)$. Hence $\delta_{x}(G)=0$.

(2) We write $\delta=\delta(G)$. If $\delta=0$, then there is nothing to show. Thus we can assume that $\delta>0$ (e.g., $G$ is non-elementary). By the compactness of $\mathbb{S}^{n-1}$, for every $\varepsilon>0$, there exists a point $x(\varepsilon) \in \mathbb{S}^{n-1}$ such that

$$
\sum_{g \in G: g(0) \in B_{r}^{n}(x(\varepsilon))} e^{-(\delta-\varepsilon) \rho(0, g(0))}=\infty \text { for all } r>0 .
$$

Let $x_{k}:=x(1 / k)$. By choosing a subsequence we can assume that $\left\{x_{k}\right\}$ converges to a point $x_{0} \in \mathbb{S}^{n-1}$. Then $x_{0}$ has the property that $\delta_{x_{0}}(G) \geq \delta$, and since $\delta_{x}(G) \leq \delta$ for each $x \in \mathbb{S}^{n-1}$, we have that $\delta_{x_{0}}(G)=\delta$.

(3) Let $x \in \mathbb{S}^{n-1}$ and assume to the contrary that $\lim _{r \rightarrow 0} \sup \left\{\delta_{w}(G) \mid w \in\right.$ $\left.\dot{B}_{r}^{n-1}(x)\right\}>\delta_{x}(G)$. Then there exists a sequence $\left\{w_{j}\right\} \subset \mathbb{S}^{n-1}$ converging to $x$, such that $\lim _{j \rightarrow \infty} \delta_{w_{j}}(G)>\delta_{x}(G)$. Write $\delta_{\infty}:=\lim _{j \rightarrow \infty} \delta_{w_{j}}(G)$ and choose $\varepsilon>0$ such that $\delta_{\infty}>\delta_{x}(G)+\varepsilon$. Let $r>0$ be arbitrary. Then for large enough $j$, we have $w_{j} \in B_{r}^{n}(x)$ and $\delta_{w_{j}}(G) \geq \delta_{x}(G)+\varepsilon$. Hence for these $j$ we have

$$
\delta_{x}^{r}(G) \geq \delta_{w_{j}}(G) \geq \delta_{x}(G)+\varepsilon .
$$

Since $\varepsilon>0$ was fixed and $r>0$ is arbitrary, this is a contradiction as $r \rightarrow 0$.

Proof of Lemma 6.5. (1) Let $x \in \mathbb{S}^{n-1} \backslash L(G)$. Then there exists an $R>0$ such that $B_{r}^{n}(x) \cap L(G)=\varnothing$ for all $r \leq R$, and so $\operatorname{dim}_{x}(L(G))=0$.

(2) By definition we have that $\operatorname{dim}_{x}(L(G)) \leq \operatorname{dim} L(G)$ for each $x \in L(G)$. Write $d=\operatorname{dim} L(G)$. Using a compactness argument one can show that there exists a point $x_{0} \in L(G)$ such that

$$
H_{d-\varepsilon}\left(B_{r}^{n}\left(x_{0}\right) \cap L(G)\right)=\infty \text { for all } \varepsilon>0 \text { and } r>0,
$$

where $H_{s}$ denotes $s$-dimensional Hausdorff measure. But this implies that $\operatorname{dim}_{x_{0}}(L(G)) \geq d$, and using the fact that $\operatorname{dim}_{x_{0}}(L(G)) \leq d$ for each $x \in$ $\mathbb{S}^{n-1}$, we conclude that $\operatorname{dim}_{x_{0}}(L(G))=d$.

(3) Let $x \in \mathbb{S}^{n-1}$.

(i) We first show that

$$
\operatorname{dim}_{x}(L(G)) \geq \lim _{r \rightarrow 0} \sup \left\{\operatorname{dim}_{w}(L(G)) \mid w \in \dot{B}_{r}^{n-1}(x)\right\} .
$$

Suppose, to the contrary, that this is not true. Then there exists a sequence $\left\{w_{j}\right\} \subset \mathbb{S}^{n-1}$ converging to $x$ such that $d_{\infty}:=\lim _{j \rightarrow \infty} \operatorname{dim}_{w_{j}}(L(G))>$ $\operatorname{dim}_{x}(L(G))$. Choose $\varepsilon>0$ such that $d_{\infty}>\operatorname{dim}_{x}(L(G))+\varepsilon$. But then arbitrarily close to $x$ the local dimension of $L(G)$ is at least $\operatorname{dim}_{x}(L(G))+\varepsilon$, and this is a contradiction. 
(ii) Next we show that

$$
\operatorname{dim}_{x}(L(G)) \leq \lim _{r \rightarrow 0} \sup \left\{\operatorname{dim}_{w}(L(G)) \mid w \in \dot{B}_{r}^{n-1}(x)\right\} .
$$

If $\operatorname{dim}_{x}(L(G))=0$, then there is nothing to show. Hence we may assume that $\operatorname{dim}_{x}(L(G))>0$, in particular, $x \in L(G)$. We will show that for every $\varepsilon>0$ and every $\delta>0$ there exists

$$
w \in\left(\dot{B}_{\delta}^{n-1}(x) \cap L(G)\right) \text { such that } \operatorname{dim}_{w}(L(G)) \geq \operatorname{dim}_{x}(L(G))-\varepsilon .
$$

This will then imply the claim.

Suppose to the contrary that (6.1) is not true. Then there exist $\varepsilon>0$ and $\delta>0$ such that $\operatorname{dim}_{w}(L(G))<\operatorname{dim}_{x}(L(G))-\varepsilon$ for all $w \in \dot{B}_{\delta}^{n-1}(x) \cap$ $L(G)$. Then for each $w \in \dot{B}_{\delta}^{n-1}(x) \cap L(G)$ there exists $r(w)>0$ such that $\operatorname{dim}\left(B_{r(w)}^{n}(w) \cap L(G)\right) \leq \operatorname{dim}_{x}(L(G))-\varepsilon / 2$. Let $s:=\operatorname{dim}_{x}(L(G))-\varepsilon / 4$. Then using the fact that $\mathbb{R}^{n}$ is second countable we can find a countable collection of balls $B_{r(w)}^{n}(w)$ that cover $\dot{B}_{\delta}^{n-1}(x) \cap L(G)$, each of which has $H_{s}$-measure 0. But this implies $\operatorname{dim}_{x}(L(G)) \leq s$, a contradiction.

Since the proof of Lemma 6.6 is a minor modification of the proof of Lemma 6.5 we will not give the details here.

Next we show that the local dimension of the conical limit set is bounded above by the local exponent of convergence.

Proof of Theorem 6.7. If $x \notin L(G)$, then $\delta_{x}(G)=\operatorname{dim}_{x}\left(L_{c}(G)\right)=0$ by Lemmas 6.3 and 6.6 Thus we only have to consider the case where $x \in L(G)$. In fact, it suffices to prove the lemma in the case where $x \in L_{c}(G)$. By using part (3) of Lemmas 6.6 and 6.3, and the fact that $L(G)$ is the closure of $L_{c}(G)$, we then conclude that the claim also holds for $x \in L(G)$.

Let $x \in L_{c}(G)$. We will show that $\operatorname{dim}_{x}\left(L_{c}(G)\right) \leq \delta_{x}^{r}(G)$ for each $r>0$. For $M>0$ and $g \in G$, let $B_{g}^{M}$ be the $n$-dimensional Euclidean ball centered at $\frac{g(0)}{|g(0)|} \in \mathbb{S}^{n-1}$ and of radius $M(1-|g(0)|)$. Define

$$
E_{M}^{r}(x):=\left\{w \in B_{r}^{n}(x) \mid w \in B_{g}^{M} \text { for infinitely many } g \in G\right\} .
$$

Then, since $G$ acts discontinuously on $\mathbb{B}^{n}$, we have that $E_{M}^{r}(x) \subset \mathbb{S}^{n-1}$, and furthermore, one easily sees that $L_{c}(G) \cap B_{r}(x)=\bigcup_{M>0} E_{M}^{r}(x)$.

Observe that $\left\{g \in G \mid \operatorname{diam}\left(B_{g}^{M}\right) \geq t\right\}$ is finite for any $t>0$. Furthermore, if $w \in B_{g}^{M} \cap B_{r}^{n}(x)$ and diam $B_{g}^{M}<\frac{2 M}{M+1} r$, then by the triangle inequality, $|g(0)-x|<$ $2 r$. Thus, if $w \in E_{M}^{r}(x)$, then $w \in B_{r}^{n}(x) \cap B_{g}^{M}$ for infinitely many $g \in G$, and so $|g(0)-x|<2 r$ for all but finitely many of these $g$. This implies that

$$
\left\{B_{g}^{M} \mid \operatorname{diam} B_{g}^{M}<t \text { and } g(0) \in B_{2 r}^{n}(x)\right\}
$$

is a cover of $E_{M}^{r}(x)$ for any $t>0$. Using this cover we will show that $\operatorname{dim} E_{M}^{r}(x) \leq$ $\delta_{x}^{2 r}(G)+\varepsilon$ for arbitrary $\varepsilon>0$.

Let $\varepsilon>0$ and define $s:=\delta_{x}^{2 r}(G)+\varepsilon$. Then

$$
\sum_{g \in G: g(0) \in B_{2 r}^{n}(x)}(1-|g(0)|)^{s}<\infty, \quad \text { and so } \sum_{g \in G: g(0) \in B_{2 r}^{n}(x)}\left(\operatorname{diam}\left(B_{g}^{M}\right)\right)^{s}<\infty .
$$


Thus, for any $t>0$,

$$
\begin{aligned}
H_{s}^{t}\left(E_{M}^{r}(x)\right) & :=\inf \left\{\sum_{j} r_{j}^{s} \mid E_{M}^{r}(x) \subset \bigcup_{r} B_{r_{j}}^{n}\left(x_{j}\right), r_{j}<t\right\} \\
& \leq \sum_{\substack{g \in G: g(0) \in B_{2 r}^{n}(x) \\
\operatorname{diam} B_{g}^{M}<t}}\left(\operatorname{diam} B_{g}^{M}\right)^{s} \leq \sum_{g \in G: g(0) \in B_{2 r}^{n}(x)}\left(\operatorname{diam} B_{g}^{M}\right)^{s} .
\end{aligned}
$$

Since the last expression is independent of $t$ and finite, we obtain that $\operatorname{dim} E_{M}^{r}(x) \leq$ $s=\delta_{x}^{2 r}(G)+\varepsilon$ for arbitrary $\varepsilon>0$. This gives $\operatorname{dim} E_{M}^{r}(x) \leq \delta_{x}^{2 r}(G)$, and it follows that $\operatorname{dim}_{x}\left(L_{c}(G)\right) \leq \delta_{x}^{2 r}(G)$ for arbitrary $r>0$.

We now briefly study the Möbius case.

Proof of Theorem 6.9. Let $\Gamma$ be a non-elementary Fuchsian group acting on $\overline{\mathbb{B}^{n}}$.

(1) Let $x \in L(\Gamma)$. Using the invariance of the hyperbolic metric on $\mathbb{B}^{n}$ under Möbius transformations we first observe that $\delta_{x}(\Gamma)=\delta_{\gamma(x)}(\Gamma)$ for all $\gamma \in \Gamma$. Let $y \in L(\Gamma)$ be arbitrary. Then there are $\gamma_{j} \in \Gamma$ such that $\gamma_{j}(x) \rightarrow y$ as $j \rightarrow \infty$. Using Lemma 6.3 we conclude that $\delta_{y}(\Gamma) \geq \lim \sup _{j \rightarrow \infty} \delta_{\gamma_{j}(x)}(\Gamma)=$ $\delta_{x}(\Gamma)$. Interchanging the roles of $x$ and $y$ we also obtain $\delta_{x}(\Gamma) \geq \delta_{y}(\Gamma)$, and we conclude that $\delta_{x}(\Gamma)$ is constant for all $x \in L(\Gamma)$. Since $\delta(\Gamma)=$ $\max _{x \in L(\Gamma)} \delta_{x}(\Gamma)$ by Lemma 6.3 we obtain that $\delta_{x}(\Gamma)=\delta(\Gamma)$ for all $x \in$ $L(\Gamma)$.

(2) Let $x \in L(\Gamma)$. Since Hausdorff dimension is preserved under Möbius maps, we obtain $\operatorname{dim}_{x}(L(\Gamma))=\operatorname{dim}_{\gamma(x)}(L(\Gamma))$ for all $\gamma \in \Gamma$. The same argument as in part (1) shows that $\operatorname{dim}_{x}(L(\Gamma))$ is constant for all $x \in L(\Gamma)$, and thus $\operatorname{dim}(L(\Gamma))=\operatorname{dim}_{x}(L(\Gamma))$ for all $x \in L(\Gamma)$.

(3) Using the invariance of the conical limit set under any $\gamma \in \Gamma$ we conclude as in part (2) that $\operatorname{dim}_{x}\left(L_{c}(\Gamma)\right)=\operatorname{dim}\left(L_{c}(\Gamma)\right)$ for all $x \in L_{c}(\Gamma)$. If $x \in L(\Gamma) \backslash L_{c}(\Gamma)$, then $\operatorname{dim}_{x}\left(L_{c}(\Gamma)\right)=\lim _{r \rightarrow 0} \sup \left\{\operatorname{dim}_{w}\left(L_{c}(\Gamma)\right) \mid w \in\right.$ $\left.\dot{B}_{r}^{n-1}(x) \cap L_{c}(\Gamma)\right\}$ by Lemma6.6. Hence the result extends to all $x \in L(\Gamma)$.

(4) It is a result in the theory of Kleinian groups (see, e.g., [3]) that $\delta(\Gamma)=$ $\operatorname{dim}\left(L_{c}(\Gamma)\right)$ for every non-elementary Kleinian group. Using parts (1) and (3), the desired result follows.

To finish this section we show that the local dimension and exponent are invariant under locally bi-Lipschitz maps.

Proof of Theorem 6.10. In the case where $w \in \mathbb{S}^{n-1} \backslash L(G)$ we have that $\delta_{w}(G)=$ $\delta_{\varphi(w)}(\widetilde{G})=0$, and so there is nothing to show; similarly for the Hausdorff dimension results. Thus we may now assume that $w \in L(G)$.

It is more convenient to work in the upper half space model. So by conjugating with a Möbius transformation, we may assume that $G$ is a discrete quasiconformal group that preserves $\mathbb{H}^{n}, \varphi$ is a quasiconformal map that preserves $\mathbb{H}^{n}$, and $\varphi$ is locally bi-Lipschitz at $w=0$.

By furthermore postcomposing $\varphi$ with a Möbius transformation, we may also assume that $\varphi(0)=0$ and $\varphi(\infty)=\infty$.

We first prove (1). Define $\psi: \mathbb{H}^{n} \rightarrow \mathbb{H}^{n}$ by

$$
\psi\left(x+t e_{n}\right):=\varphi(x)+e_{n} \cdot \max \left\{|\varphi(y)-\varphi(x)|\left|y \in \mathbb{R}^{n-1},\right| y-x \mid=t\right\}
$$


for $x \in \mathbb{R}^{n-1} \subset \mathbb{R}^{n}$ and $t>0$ (here, $e_{n}$ denotes the $n$th unit vector in $\mathbb{R}^{n}$ ). Then for any $z \in \mathbb{H}^{n}$ we have $\rho(\varphi(z), \psi(z)) \leq C(n, K)$, where $C(n, K)$ depends only on $n$ and the quasiconformal dilatation $K$ of $\varphi$, but not on $\varphi$ itself or $z$ (see, for example, [27] together with Lemma 3.1 in [5]). Furthermore, by the bi-Lipschitz assumption, we have that $t / L \leq|\varphi(y)-\varphi(x)| \leq L t$ for all $x, y \in B_{r}(0) \cap \mathbb{R}^{n-1}$ with $|x-y|=t$. Hence

$$
\rho\left(\varphi\left(x+t e_{n}\right), \varphi(x)+t e_{n}\right) \leq C(n, K)+\log L=: C
$$

for all $x \in B_{r / 2}(0) \cap \mathbb{R}^{n-1}$ and $t<r / 2$. For such $x$ and $t$ we have

$$
\begin{aligned}
\rho\left(e_{n}, \varphi\left(x+t e_{n}\right)\right) & \leq \rho\left(e_{n}, e_{n}+\varphi(x)\right)+\rho\left(\varphi(x)+e_{n}, \varphi(x)+t e_{n}\right)+C \\
& \leq \rho\left(e_{n}, e_{n}+\frac{L r}{2} e_{1}\right)+\rho\left(e_{n}, t e_{n}\right)+C .
\end{aligned}
$$

In the same way we obtain $\rho\left(e_{n}, t e_{n}\right) \leq \rho\left(e_{n}, \varphi\left(x+t e_{n}\right)\right)+\widetilde{C}$ for some constant $\widetilde{C}$ that only depends on $L, r, n, K$, so that $\rho\left(e_{n}, \varphi\left(x+t e_{n}\right)\right) \sim \rho\left(e_{n}, t e_{n}\right)$ for all $x \in B_{r / 2}(0) \cap \mathbb{R}^{n-1}$ and $t<r / 2$. This implies $\delta_{0}\left(\varphi G \varphi^{-1}\right)=\delta_{0}(G)$.

For the proofs of parts (2) and (3) observe that $\varphi(L(G))=L(\widetilde{G}), \varphi\left(L_{c}(G)\right)=$ $L_{c}(\widetilde{G})$, and the Hausdorff dimension is invariant under bi-Lipschitz maps; hence the local Hausdorff dimension is invariant under locally bi-Lipschitz maps.

\section{The Support of Patterson-Sullivan Measures}

Let $G$ be a discrete QCF group acting on $\overline{\mathbb{B}^{n}}$, and let $\delta=\delta(G)$. We have seen in the construction of the Patterson-Sullivan measure $\mu_{x, y}^{\delta}\left(\right.$ for $x, y \in \mathbb{B}^{n}$ ) that this measure is supported on the limit set $L(G)$. Using the local exponent of convergence as defined in Section [6] we can be more precise. Recall that, for any measure $\mu$ on $\mathbb{S}^{n-1}$, its support $\operatorname{supp}(\mu)$ is defined as the smallest closed set $F$ in $\mathbb{S}^{n-1}$ such that $\mu(E)=0$ for any measurable set $E \subset \mathbb{S}^{n-1} \backslash F$.

Theorem 7.1. Let $G$ be a discrete $Q C F$ group acting on $\overline{\mathbb{B}^{n}}$, and $\delta$ its exponent of convergence. For any $x, y \in \mathbb{B}^{n}$ we then have that

$$
\operatorname{supp}\left(\mu_{x, y}^{\delta}\right) \subset\left\{\zeta \in \mathbb{S}^{n-1} \mid \delta_{\zeta}(G)=\delta\right\}
$$

Proof of Theorem 7.1. Observe first that $F:=\left\{\zeta \in \mathbb{S}^{n-1} \mid \delta_{\zeta}(G)=\delta\right\}$ is a closed set. For if $\zeta_{k} \in F$ and $\zeta_{k} \rightarrow \zeta$, then $\delta_{\zeta}(G) \geq \lim \sup _{k \rightarrow \infty} \delta_{\zeta_{k}}(G)=\delta$ by Lemma 6.3. Since, on the other hand, $\delta_{\zeta}(G) \leq \delta$, we conclude that $\zeta \in F$.

Let $x, y \in \mathbb{B}^{n}$ and let $\mu_{x, y}^{\delta}$ be the weak limit of the measures $\mu_{x, y}^{s_{k}}$ for some sequence $s_{k} \rightarrow \delta^{+}$.

For $j \in \mathbb{N}$, define $U_{j}:=\left\{z \in \overline{\mathbb{B}^{n}} \mid \operatorname{dist}(z, F)>\frac{1}{j}\right\}$. Then $U_{j}$ is relatively open in $\overline{\mathbb{B}^{n}}$, and hence by the weak convergence of the measures,

$$
\mu_{x, y}^{\delta}\left(U_{j}\right) \leq \liminf _{k \rightarrow \infty} \mu_{x, y}^{s_{k}}\left(U_{j}\right)
$$

Let $d_{j}:=\sup \left\{\delta_{z}(G) \mid z \in U_{2 j} \cap \mathbb{S}^{n-1}\right\}$. Then $d_{j}<\delta$ since $\overline{U_{2 j} \cap \mathbb{S}^{n-1}} \cap F=\varnothing$. We will show that for each $j$ we have $\mu_{x, y}^{s_{k}}\left(\overline{U_{j}}\right) \rightarrow 0$ as $k \rightarrow \infty$. First we note that

$$
\mu_{x, y}^{s_{k}}\left(\overline{U_{j}}\right) \leq \frac{1}{\Sigma^{*}\left(s_{k}, y, y\right)} \sum_{g \in G: g(y) \in \overline{U_{j}}} e^{-\delta \rho(x, g(y))} h\left(e^{\rho(x, g(y))}\right) .
$$


Next, observe that by compactness of $\overline{U_{j}}$ we can find finitely many $z_{1}, \ldots, z_{m} \in U_{j}$ such that $\overline{U_{j}} \subset B_{1 /(2 j)}\left(z_{1}\right) \cup \ldots \cup B_{1 /(2 j)}\left(z_{m}\right)$. But for each such $z_{l}$ we have $\delta_{z_{l}}^{1 /(2 j)}(G) \leq d_{j}<\delta$, and hence we conclude

$$
\sum_{g \in G: g(y) \in \overline{U_{j}}} e^{-\delta \rho(x, g(y))} h\left(e^{\rho(x, g(y))}\right)<\infty .
$$

On the other hand, we have $\Sigma^{*}\left(s_{k}, y, y\right) \rightarrow \infty$ as $k \rightarrow \infty$, so that $\mu_{x, y}^{s_{k}}\left(\overline{U_{j}}\right) \rightarrow 0$ as $k \rightarrow \infty$. Hence $\mu_{x, y}\left(U_{j}\right)=0$ for all $j$, and so $\mu_{x, y}\left(\mathbb{S}^{n-1} \backslash F\right)=0$.

\section{Atoms for Patterson-Sullivan Measures}

We are now ready to construct examples. The fundamental relationship between the exponent of convergence and the Hausdorff dimension is that the exponent of convergence is an upper bound for the dimension of the conical limit set (Theorem 3.8). Our first example shows that the exponent of convergence of a discrete QCF group can be strictly larger than the dimension of the group's conical limit set, as opposed to in the Kleinian case. The example furthermore shows that the local exponent of convergence can be strictly larger at a point than the supremum over the local exponents of convergence in a neighborhood of that point (compare (3) in Lemma 6.3).

Example 8.1. There exists a discrete QCF group $G$ acting on $\overline{\mathbb{B}^{2}}$ such that $\delta_{x_{0}}(G)$ $>\sup \left\{\delta_{w}(G) \mid w \in L(G) \backslash\left\{x_{0}\right\}\right\}$ for some $x_{0} \in L(G)$. Furthermore, $\operatorname{dim} L_{c}(G)<$ $\delta(G)$, and for all $x, y \in \mathbb{B}^{2}$ the Patterson-Sullivan measure $\mu_{x, y}^{\delta}$ has an atom at $x_{0}$.

Construction. It will be more convenient to work in the upper half-space model $\mathbb{H}^{2}$. Let $\Gamma$ be a Fuchsian group acting on $\mathbb{H}^{2}$ which contains a parabolic element with fixed point at $\infty$, and which furthermore satisfies $\delta(\Gamma)<1$. We can assume, by conjugation, that $\gamma(z)=z+1$ is a generator of $\Gamma$. Choose $K>1$ so large that $\frac{K}{K+1}>\delta(\Gamma)$, and define $\varphi: \overline{\mathbb{R}^{2}} \rightarrow \overline{\mathbb{R}^{2}}$ by

$$
\varphi(z):= \begin{cases}z & \text { if }|z| \leq 1 \\ |z|^{\frac{1}{K}-1} z & \text { if }|z|>1\end{cases}
$$

Let $G=\varphi \circ \Gamma \circ \varphi^{-1}$. One easily verifies that, for $n \in \mathbb{Z}$, we have

$$
\rho\left(i, \varphi \gamma^{n} \varphi^{-1}(i)\right)=\rho(i, \varphi(i+n)) \sim \log \left(|n|^{\frac{1}{K}+1}\right),
$$

where " " means that the two quantities only differ by an additive constant which is bounded independently of $n$, and $i=(0,1) \in \mathbb{R}^{2}$. Hence

$$
\delta_{\infty}(G) \geq \delta_{\infty}\left(\left\langle\varphi \gamma \varphi^{-1}\right\rangle\right)=\frac{K}{K+1}>\delta(\Gamma) .
$$

On the other hand, $\varphi$ is locally bi-Lipschitz at every $x \in \mathbb{R}$ (but not at $\infty$ ) and so $\delta_{x}(\Gamma)=\delta_{\varphi(x)}(G)$ for every $x \in \mathbb{R}$ (Theorem [6.10). Using Theorem 6.9 we thus obtain $\delta_{w}(G)=\delta(\Gamma)$ for every $w \in L(G) \backslash\{\infty\}$. Furthermore we have $\operatorname{dim}_{x}\left(L_{c}(\Gamma)\right)=\operatorname{dim}_{\varphi(x)}\left(L_{c}(G)\right)$ for every $x \in \mathbb{R} ;$ hence $\operatorname{dim}_{w}\left(L_{c}(G)\right)=\operatorname{dim}\left(L_{c}(\Gamma)\right.$ for all $w \in L(G) \backslash\{\infty\}$. Using Lemma 6.6(2) we conclude that $\operatorname{dim}_{\infty}\left(L_{c}(G)\right)=$ $\operatorname{dim}\left(L_{c}(\Gamma)\right.$ as well, so that

$$
\operatorname{dim}\left(L_{c}(G)\right)=\operatorname{dim}\left(L_{c}(\Gamma)\right.
$$


On the other hand,

$$
\delta(G)=\delta_{\infty}(G)>\delta_{w}(G)=\delta(\Gamma)=\operatorname{dim}\left(L_{c}(\Gamma)=\operatorname{dim}\left(L_{c}(G)\right)\right.
$$

for all $w \in L(G) \backslash\{\infty\}$.

Finally, using Theorem 7.1 we find that for any $x, y \in \mathbb{H}^{2}$, the Patterson-Sullivan measure $\mu_{x, y}^{\delta}$ is supported on the set $\{\infty\}$, and so it has an atom at $\infty$.

Remark 8.1. If $\Gamma$ is a finitely generated Fuchsian group acting on $\overline{\mathbb{B}^{2}}$ with non-empty regular set, then $\delta(\Gamma)<1$.

In the above example, the atom is a parabolic fixed point. One can modify the example as follows, so that the Patterson-Sullivan measure has an atom on a conical limit point.

Example 8.2. There exists a discrete QCF group $G$ acting on $\overline{\mathbb{B}^{2}}$, such that $\delta_{x}(G)<\delta(G)$ for all $x \in L(G) \backslash\left\{x_{0}\right\}$. Furthermore $\delta_{x_{0}}(G)=\delta(G)$, where $x_{0}$ is a conical limit point of $G$.

We will use two auxiliary results in order to construct the group in the example. The proofs of these results are straightforward and we will only give their outlines.

Throughout the remainder of this section, for any $K \geq 1$, we will denote by $\varphi_{K}: \overline{\mathbb{R}^{2}} \rightarrow \overline{\mathbb{R}^{2}}$ the radial quasiconformal mapping given by

$$
\varphi_{K}(z):=|z|^{\frac{1}{K}-1} z .
$$

Proposition 8.2. Let $G$ be a discrete quasiconformal group acting on $\mathbb{H}^{2}$ with $0 \in L(G)$, and let $\beta \in M \ddot{b} b\left(\mathbb{H}^{2}\right)$. For arbitrary $R>0$ define $\tau \in M \ddot{o} b\left(\mathbb{H}^{2}\right)$ by $\tau(x):=R x+\beta(0)$, and set $\hat{\varphi}_{K}:=\tau \varphi_{K} \tau^{-1}$. Then

$$
\delta_{0}\left(\varphi_{K} G \varphi_{K}^{-1}\right)=\delta_{\beta(0)}\left(\hat{\varphi}_{k} \beta G \beta^{-1} \hat{\varphi}_{K}^{-1}\right) .
$$

Proof of Proposition 8.2. It is easy to see that for a discrete quasiconformal group $G$ acting on $\mathbb{H}^{2}$ and any $x \in \mathbb{R}$, one can compute the local exponent of convergence at $x$ by

$$
\delta_{x}(G)=\lim _{r \rightarrow 0} \inf \left\{s>0 \mid \sum_{g(i) \in B_{r}(x)}(\operatorname{Im} g(i))^{s}<\infty\right\} .
$$

The key part in proving the proposition then consists in showing that if $\alpha$ is a Möbius transformation of $\mathbb{H}^{2}$ that keeps 0 invariant, then

$$
\delta_{0}\left(\varphi_{K} G \varphi_{K}^{-1}\right)=\delta_{0}\left(\varphi_{K} \alpha G \alpha^{-1} \varphi_{K}^{-1}\right) .
$$

This is shown by observing that $\alpha(z) \approx c z$ for $z$ near 0 , where $c=\alpha^{\prime}(0)>0$. Hence

$$
\varphi_{K} \alpha(z) \approx c^{1 / K} \varphi_{K}(z) \text { for } z \text { near } 0,
$$

and so $\operatorname{Im}\left(\varphi_{K} \alpha(z)\right) \approx c^{1 / K} \operatorname{Im}\left(\varphi_{K}(z)\right)$, and this proves [8.1).

Lemma 8.3. Let $\Gamma$ be a non-elementary Fuchsian group acting on $\mathbb{H}^{2}$ with $0 \in$ $L(\Gamma)$. If there exists $K_{0}>1$ such that $\delta_{0}\left(\varphi_{K_{0}} \Gamma \varphi_{K_{0}}^{-1}\right)>\delta_{0}(\Gamma)$, then for any $K>K_{0}$ we have

$$
\delta_{0}\left(\varphi_{K} \Gamma \varphi_{K}^{-1}\right)>\delta_{0}\left(\varphi_{K_{0}} \Gamma \varphi_{K_{0}}^{-1}\right)
$$


Proof of Lemma 8.3. Write $G_{K}:=\varphi_{K} \circ \Gamma \circ \varphi_{K}^{-1}$. Let $s:=\frac{1}{2}\left(\delta_{0}(\Gamma)+\delta_{0}\left(G_{K_{0}}\right)\right)>$ $\delta_{0}(\Gamma)$, and choose $\varepsilon_{0}>0$ arbitrary such that $\delta_{0}(\Gamma)<s<s+\varepsilon_{0}<\delta_{0}\left(G_{K_{0}}\right)$. Then, for all $r>0$,

$$
\sum_{\gamma \in \Gamma: \gamma(i) \in B_{r}(0)}(\operatorname{Im} \gamma(i))^{s}<\infty \quad \text { and } \sum_{\gamma \in \Gamma: \gamma(i) \in B_{r}(0)}\left(\operatorname{Im} \varphi_{K_{0}}(\gamma(i))\right)^{s+\varepsilon_{0}}=\infty .
$$

Setting

$$
M_{r}^{\varepsilon_{0}}:=\left\{\gamma \in \Gamma \mid \gamma(i) \in B_{r}(0) \text { and }\left(\operatorname{Im} \varphi_{K_{0}}(\gamma(i))\right)^{s+\varepsilon_{0}} \geq(\operatorname{Im} \gamma(i))^{s}\right\}
$$

we conclude that $M_{r}^{\varepsilon_{0}}$ is an infinite set, and furthermore

$$
\sum_{\gamma \in M_{r}^{\varepsilon_{0}}}\left(\operatorname{Im} \varphi_{K_{0}}(\gamma(i))\right)^{s+\varepsilon_{0}}=\infty \quad \text { and } \sum_{\gamma \in \Gamma: \gamma(i) \in B_{r}(0), \gamma \notin M_{r}^{\varepsilon_{0}}}\left(\operatorname{Im} \varphi_{K_{0}}(\gamma(i))\right)^{s+\varepsilon_{0}}<\infty .
$$

Writing $\gamma(i)=\rho e^{i \theta}, 0<\theta<\pi$, for $\gamma \in M_{r}^{\varepsilon_{0}}$, one obtains

$$
\sin \theta \geq \rho^{\left(s\left(K_{0}-1\right)-\varepsilon_{0}\right) /\left(K_{0} \varepsilon_{0}\right)},
$$

and from the theory of quasiconformal mappings it follows that $\delta_{0}\left(G_{K_{0}}\right) \leq K_{0} \delta_{0}(\Gamma)$, so that the exponent of $\rho$ in (8.2) is positive.

Let $K>K_{0}$. One can now show that there exists $\varepsilon>0$, independent of $r$ and $\varepsilon_{0}$, such that

$$
\left(\rho^{1 / K_{0}} \sin \theta\right)^{s+\varepsilon_{0}} \leq\left(\rho^{1 / K} \sin \theta\right)^{s+\varepsilon_{0}+\varepsilon}
$$

for all $\gamma \in M_{r}^{\varepsilon_{0}}$, and hence $\sum_{\gamma \in M_{r}^{\varepsilon_{0}}}\left(\operatorname{Im} \varphi_{K}(\gamma(i))\right)^{s+\varepsilon_{0}+\varepsilon}=\infty$. (In fact, one can choose

$$
\left.\varepsilon=s \frac{K-K_{0}}{K\left[\frac{\delta_{0}\left(G_{K_{0}}\right)+\delta_{0}(\Gamma)}{\delta_{0}\left(G_{K_{0}}\right)-\delta_{0}(\Gamma)}\left(K_{0}-1\right)-1\right]+K_{0}} .\right)
$$

This implies that $\delta_{0}\left(G_{K}\right) \geq s+\varepsilon_{0}+\varepsilon$, where $\varepsilon>0$ is independent of $\varepsilon_{0}$. But $\varepsilon_{0}$ was chosen arbitrarily with $s+\varepsilon_{0}<\delta_{0}\left(G_{K_{0}}\right)$, and we obtain $\delta_{0}\left(G_{K}\right) \geq \delta_{0}\left(G_{K_{0}}\right)+\varepsilon$.

We are now ready to outline the construction of the group $G$ in example 8.2 .

Construction. Let $\Gamma$ be a non-elementary, finitely generated Fuchsian group acting on $\mathbb{H}^{2}$ that contains a parabolic element, and that satisfies $\delta(\Gamma)<1$. Choose $\infty \neq x_{0} \in L_{c}(\Gamma)$ and furthermore let $w_{0} \in L(\Gamma)$ be a parabolic fixed point. Choose $\gamma_{n} \in \Gamma$ such that $\infty \neq w_{n}:=\gamma_{n}\left(w_{0}\right) \rightarrow x_{0}$. Choose $K>2$ such that $\frac{K}{K+1}>\delta(\Gamma)$, and set $K_{n}:=K-1 / n$. Inductively, we now choose radii $r_{n}>0$ such that the disks $\left\{B_{2 r_{n}}\left(w_{n}\right)\right\}$ are mutually disjoint, and furthermore satisfy $\sum r_{n}^{\delta(\Gamma) / K_{n}}<1$. Then we define the mapping $\varphi$ that conjugates $\Gamma$ to the desired quasiconformal group $G$ as follows:

$$
\varphi(z)= \begin{cases}r_{n} \varphi_{K_{n}}\left(\frac{z-w_{n}}{r_{n}}\right)+w_{n} & \text { if } z \in B_{r_{n}}\left(w_{n}\right), \\ z & \text { otherwise } .\end{cases}
$$

One easily verifies that $\varphi$ is indeed a $K$-quasiconformal mapping. Now let $G=$ $\varphi \Gamma \varphi^{-1}$. Then $L(G)=\varphi(L(\Gamma))$, and for any $\varepsilon>0$, the mapping $\varphi$ has a uniformly bounded derivative on $\mathbb{R} \backslash \bigcup B_{\varepsilon r_{n}}\left(w_{n}\right)$. Since furthermore $\sum r_{n}^{\delta(\Gamma) / K_{n}}<1$, one can 
show that $\operatorname{dim} L(G)=\operatorname{dim} L(\Gamma)$. As in Example 8.1 and using Lemma 6.3, one furthermore sees that

$$
\begin{aligned}
\delta_{w_{n}}(G) & \geq \frac{K_{n}}{K_{n}+1} \text { for all } n \\
\delta_{x_{0}}(G) & \geq \limsup _{n \rightarrow \infty} \delta_{w_{n}}(G) \text { and } \\
\delta_{x}(G) & =\delta(\Gamma) \text { for all other } x \in L(G) .
\end{aligned}
$$

Using Proposition 8.2 and Lemma 8.3 we now obtain that $\delta_{w_{n+1}}(G)>\delta_{w_{n}}(G)>$ $\delta(\Gamma)$ for large enough $n$, so that $\delta_{x_{0}}(G)>\delta_{x}(G)$ for all $x \in L(G) \backslash\left\{x_{0}\right\}$.

\section{QuASI-INVARIANCE}

We have seen that, in general, the exponent of convergence of a discrete QCF group is not equal to the Hausdorff dimension of its conical limit set.

In this section we provide a sufficient condition on the behavior of PattersonSullivan measure under the action of the group so that the Hausdorff dimension of the conical limit set does equal the exponent of convergence.

We first fix some notation. Let $G$ be a discrete QCF group acting on $\overline{\mathbb{B}^{n}}$. For a point $\zeta \in \mathbb{S}^{n-1}$ and a base point $x \in \mathbb{B}^{n}$ let $h_{\zeta, x}(t) \in \mathbb{B}^{n}$ denote the point at hyperbolic distance $t$ from $x$ on the directed geodesic from $x$ to $\zeta$. Furthermore, let $\sigma_{\zeta, x}(t):=\min \left\{\rho\left(h_{\zeta, x}(t), g(x)\right) \mid g \in G\right\}$ be the distance of $h_{\zeta, x}(t)$ to the orbit of $x$. We can now formulate the main theorem of this section.

Theorem 9.1. Let $G$ be a discrete $Q C F$ group on $\overline{\mathbb{B}^{n}}$ such that for fixed $x \in \mathbb{B}^{n}$ there exists a constant $C_{x}<\infty$ so that $\sigma_{\zeta, x}(t)<C_{x}$ for all $\zeta \in L(G)$ and all $t>0$. Assume furthermore that there exists a constant $A>0$ such that

$$
\mu_{g^{-1}(x), y}^{\delta} \leq A \cdot g * \mu_{x, y}^{\delta}
$$

for all $x, y \in \mathbb{B}^{n}$ and all $g \in G$.

Then $\delta_{z}(G)=\operatorname{dim}_{z} L(G)=\delta(G)=\operatorname{dim} L(G)$ for all $z \in L(G)$.

Remark 9.2.

- The first condition above means that the limit points of $G$ are uniformly conical. In [4] we show that this condition is satisfied, for example, in the case of a discrete QCF group with purely conical limit set.

- If $G$ satisfies (9.1), then it necessarily satisfies

$$
\frac{1}{A} \cdot g * \mu_{x, y}^{\delta} \leq \mu_{g^{-1}(x), y}^{\delta} \leq A \cdot g * \mu_{x, y}^{\delta}
$$

for all $x, y \in \mathbb{B}^{n}$ and all $g \in G$.

Examples for QCF groups which satisfy the assumptions of the above theorem are given by the following result.

Theorem 9.3. Let $\Gamma$ be a convex-compact Kleinian group acting on $\mathbb{H}^{n}$, and let $\varphi: \mathbb{R}^{n-1} \rightarrow \mathbb{R}^{n-1}$ be a bi-Lipschitz boundary mapping with $\varphi(\infty)=\infty$. Let $\varphi$ also denote a quasiconformal extension of this boundary mapping to $\mathbb{H}^{n}$, and define $G:=\varphi \Gamma \varphi^{-1}$. Then $G$ satisfies the assumptions of Theorem 9.1.

We need two geometric facts in the proof of Theorem 9.1 
Lemma 9.4. Let $z \in \mathbb{B}^{n}$ and let $C_{0} \geq 1$ be a constant. Then there exist constants $0<C<1$ and $\rho_{0}>0$ depending only on $z$ and $C_{0}$ such that the following is true: For any $w \in \mathbb{B}^{n}$ with $\rho(z, w) \geq \rho_{0}$, let $\zeta_{0} \in \mathbb{S}^{n-1}$ be the endpoint of the directed hyperbolic ray starting at $z$ and going through $w$. Then

$$
C e^{\rho(z, w)} \leq \frac{P(w, \zeta)}{P(z, \zeta)} \leq e^{\rho(z, w)}
$$

for any $\zeta \in B_{C_{0} e^{-\rho\left(w_{0}, z\right)}}\left(\zeta_{0}\right) \cap \mathbb{S}^{n-1}$.

Lemma 9.5. For every $\varepsilon>0$ there exist constants $C_{0} \geq 1$ and $\rho_{0}>0$ such that the following is true: Let $z \in \mathbb{B}^{n}$ and $\zeta_{0} \in \mathbb{S}^{n-1}$. If $w \in \mathbb{B}^{n}$ with $\rho(z, w) \geq \rho_{0}$, and $w$ has hyperbolic distance at most $\varepsilon$ from the hyperbolic ray starting at $z$ and ending at $\zeta_{0}$, then

$$
B_{C_{0} e^{-\rho(z, w)}}(\zeta) \supset B_{e^{-\rho(z, w)}}\left(\zeta_{0}\right),
$$

where $\zeta$ is the endpoint of the directed hyperbolic ray starting at $z$ and going through $w$.

Since the proofs of these lemmas are straightforward, we omit them.

Proof of Theorem 9.1. Fix $x, y \in \mathbb{B}^{n}$. By assumption, there exists a constant $C_{x}>$ 0 such that each point on any hyperbolic ray starting at $x$ and ending at a point $\zeta \in L(G)$ has at most hyperbolic distance $C_{x}$ from the orbit of $x$. Following Lemma 9.5, there exist constants $C_{0} \geq 1$ and $\widetilde{\rho}_{0}>0$ depending only on $C_{x}$ such that the following is true: For any $\zeta_{0} \in L(G)$, and any $g \in G$ such that $\rho(x, g(x)) \geq \widetilde{\rho}_{0}$, and such that the hyperbolic distance between $g(x)$ and the hyperbolic ray from $x$ to $\zeta_{0}$ is at most $C_{x}$, we have

$$
B_{C_{0} e^{-\rho(x, g(x))}}(\zeta) \supset B_{e^{-\rho(x, g(x))}}\left(\zeta_{0}\right)
$$

where $\zeta$ is the endpoint of the directed hyperbolic ray starting at $x$ and passing through $g(x)$.

By Lemma 9.4, there now exist constants $0<C<1$ and $\rho_{0}>\widetilde{\rho}_{0}$ depending only on $C_{0}$ and $x$ such that

$$
C e^{\rho(x, g(x))} \leq \frac{P(g(x), \zeta)}{P(x, \zeta)} \leq e^{\rho(x, g(x))}
$$

for all $g \in G$ that satisfy $\rho(x, g(x)) \geq \rho_{0}$ and all $\zeta \in B_{C_{0} e^{-\rho(x, g(x))}}\left(\zeta_{0}\right) \cap \mathbb{S}^{n-1}$, where $\zeta_{0}$ is the endpoint of the directed hyperbolic ray starting at $x$ and passing through $g(x)$.

Now let $g \in G$ with $\rho(x, g(x)) \geq \rho_{0}$. Set $r_{g}=\rho(x, g(x))$, and let $\zeta_{g}$ be the endpoint of the directed hyperbolic ray starting at $x$ and passing through $g(x)$. Using Lemma 4.1 and (9.3) we obtain that

$$
\begin{aligned}
\mu_{g(x), y}^{\delta}\left(B_{C_{0} r_{g}}\left(\zeta_{g}\right)\right) & =\int_{B_{C_{0} r_{g}}\left(\zeta_{g}\right)}\left(\frac{P(g(x), \zeta)}{P(x, \zeta)}\right)^{\delta} \mu_{x, y}^{\delta}(d \zeta) \\
& \geq C^{\delta} e^{\delta \rho(x, g(x))} \mu_{x, y}^{\delta}\left(B_{C_{0} r_{g}}\left(\zeta_{g}\right)\right)=C_{0}^{\delta} C^{\delta} \frac{\mu_{x, y}^{\delta}\left(B_{C_{0} r_{g}}\left(\zeta_{g}\right)\right)}{\left(C_{0} r_{g}\right)^{\delta}}
\end{aligned}
$$


Using assumption (9.1) we conclude from this that

$$
\begin{aligned}
\frac{\mu_{x, y}^{\delta}\left(B_{C_{0} r_{g}}\left(\zeta_{g}\right)\right)}{\left(C_{0} r_{g}\right)^{\delta}} & \leq\left(C_{0} C\right)^{-\delta} A \mu_{x, y}^{\delta}\left(g^{-1}\left(B_{C_{0} r_{g}}\left(\zeta_{g}\right)\right)\right) \\
& \leq\left(C_{0} C\right)^{-\delta} A \mu_{x, y}^{\delta}\left(\mathbb{S}^{n-1}\right)=: \widetilde{C}
\end{aligned}
$$

Next we show that there exists a constant $D<\infty$ so that

$$
\frac{\mu_{x, y}\left(B_{r}(\zeta)\right)}{r^{\delta}} \leq D
$$

holds for all $\zeta \in L(G)$ and all sufficiently small $r>0$.

Let $\zeta \in L(G)$. Inductively, using the assumption that $\sigma_{\zeta, x}(t)<C_{x}$ for all $\zeta \in$ $L(G)$ and all $t>0$, we can choose $g_{i} \in G$ for $i=0,1,2, \ldots$ such that $\rho\left(x, g_{0}(x)\right) \leq$ $\rho_{0}+2 C_{x}, \rho\left(x, g_{i}(x)\right) \geq \rho_{0}$ for all $i$, and furthermore $g_{i}(x) \rightarrow \zeta, \rho\left(g_{i}(x), g_{x, \zeta}\right) \leq C_{x}$, and $\rho\left(g_{i}(x), g_{i+1}(x)\right) \leq 5 C_{x}$.

For each $i \in \mathbb{N}$, let $\zeta_{i}$ be the endpoint of the directed hyperbolic ray from $x$ through $g_{i}(x)$, and let $r_{i}=e^{-\rho\left(x, g_{i}(x)\right)}$. Using (9.2) and (9.4) we obtain that

$$
\frac{\mu_{x, y}^{\delta}\left(B_{r_{i}}(\zeta)\right)}{r_{i}^{\delta}} \leq \frac{\mu_{x, y}^{\delta}\left(B_{C_{0} r_{i}}\left(\zeta_{i}\right)\right)}{\left(C_{0} r_{i}\right)^{\delta}} C_{0}^{\delta} \leq \widetilde{C} C_{0}^{\delta} .
$$

Now let $0<r<e^{-\rho_{0}-2 C_{x}}=: r^{*}$ be arbitrary. Since $r^{*} \leq r_{0}$, there exists $i \in \mathbb{N}$ such that $r_{i+1} \leq r<r_{i}$, and so

$$
\frac{\mu_{x, y}^{\delta}\left(B_{r}(\zeta)\right)}{r^{\delta}} \leq \frac{\mu_{x, y}^{\delta}\left(B_{r_{i}}(\zeta)\right)}{r_{i}^{\delta}} \frac{r_{i}^{\delta}}{r_{i+1}^{\delta}} \leq \widetilde{C} C_{0}^{\delta} e^{\delta 5 C_{x}}=: D
$$

and this shows that (9.5) holds.

Inequality (9.5) now enables us to show that the Hausdorff dimension of $G$ 's limit set is at least $\delta$ : let $\bigcup B_{i}$ be any covering of $L(G)$ with balls centered on $L(G)$ of radius $s_{i} \leq r^{*}$. Then

$$
\sum_{i} s_{i}^{\delta} \geq D \sum_{i} \mu_{x, y}^{\delta}\left(B_{i}\right) \geq D \mu_{x, y}^{\delta}\left(\bigcup B_{i}\right) \geq D \mu_{x, y}^{\delta}(L(G)) .
$$

Since $\mu_{x, y}^{\delta}(L(G))>0$, we conclude that the Hausdorff $\delta$-measure of $L(G)$ is nonzero, and hence the Hausdorff dimension of $L(G)$ must be at least $\delta$. Since, on the other hand, $\operatorname{dim} L(G) \leq \delta(G)$ by Theorem 3.8, we have equality of $\operatorname{dim} L(G)$ and $\delta(G)$.

To see that $\delta_{z}(G)=\delta(G)$ for all $z \in L(G)$, assume that this were not the case. Then, using (3) of Lemma 6.3 we find $z \in L(G)$ and $r>0$ such that $\delta_{w}(G)<\delta(G)$ for all $w \in B_{r}(z)$. Now Theorem[7.1 implies that $\mu_{x, y}^{\delta}\left(B_{r}(z)\right)=0$. Choose $g_{1}, g_{2} \in G$ such that $g_{1}\left(B_{r}(z)\right) \cup g_{2}\left(B_{r}(z)\right) \supset L(G)$. Using assumption (9.1) we obtain for $i=1,2$ that $\mu_{g_{i}(x), y}^{\delta}\left(g_{i}\left(B_{r}(z)\right)\right)=0$, and since $\mu_{g_{i}(x), y}^{\delta}$ and $\mu_{x, y}^{\delta}$ are mutually absolutely continuous we conclude that $\mu_{x, y}^{\delta}\left(g_{i}\left(B_{r}(z)\right)\right)=0$. But this implies that $\mu_{x, y}^{\delta}(L(G))=0$, a contradiction.

Finally, the same argument that was used to show that the Hausdorff dimension of $L(G)$ is at least $\delta$ can be used to show that the Hausdorff $\delta$-measure of $L(G) \cap$ $B_{r}(\zeta)$ is bounded below by a constant times $\mu_{x, y}^{\delta}\left(L(G) \cap B_{r}(\zeta)\right)$, and this shows that $\mu_{x, y}^{\delta}$ is absolutely continuous with respect to the $\delta$-dimensional Hausdorff measure on $L(G)$. As above, using the assumption (9.1), one sees that $\mu_{x, y}^{\delta}\left(L(G) \cap B_{r}(\zeta)\right)>$ 0 , and this implies that $\operatorname{dim} L(G) \cap B_{r}(\zeta) \geq \delta$ for all $\zeta \in L(G)$ and all $r>0$. 
Hence $\operatorname{dim}_{\zeta}(L(G)) \geq \delta(G)=\delta_{\zeta}(G)$. Since, on the other hand, $\operatorname{dim}_{\zeta}(L(G)) \leq$ $\operatorname{dim}(L(G))=\delta(G)$, we obtain equality of all these quantities.

We now show that Theorem 9.1 is not vacuously satisfied in the class of discrete QCF groups. The key geometric idea shown in the proof of this theorem is that any quasiconformal extension to $\mathbb{H}^{n}$ of a bi-Lipschitz mapping on $\mathbb{R}^{n-1}$ is a $(1, C)$ pseudo-isometry with respect to the hyperbolic metric.

Proof of Theorem 9.3. Observe that every bi-Lipschitz map is quasiconformal, where the dilatation only depends on the Lipschitz constant and on the dimension $n$. Thus $\varphi$ is quasiconformal on $\mathbb{R}^{n-1}$ and can be extended to a quasiconformal mapping of $\mathbb{H}^{n}$ whose dilatation only depends on its Lipschitz constant $L$ and on the dimension $n$ (we could have chosen any quasiconformal extension here). By abuse of notation we denote the extension by $\varphi$ again.

Recall that $\delta(G)=\delta(\Gamma)$ by Theorem 6.10

We first show that there exists a constant $D=D(L, n)>0$ such that for all $z, w \in \mathbb{H}^{n}$ we have

$$
|\rho(\varphi(z), \varphi(w))-\rho(z, w)| \leq D
$$

As in the proof of Theorem 6.10, we define a map $\psi: \mathbb{H}^{n} \rightarrow \mathbb{H}^{n}$ by

$$
\psi\left(x+t e_{n}\right):=\varphi(x)+e_{n} \cdot \max \left\{|\varphi(y)-\varphi(x)|\left|y \in \mathbb{R}^{n-1},\right| y-x \mid=t\right\}
$$

for $x \in \mathbb{R}^{n-1} \subset \mathbb{R}^{n}$ and $t>0$. Then for any $z \in \mathbb{H}^{n}$ we have $\rho(\varphi(z), \psi(z)) \leq$ $C(n, L)$, where $C(n, L)$ depends only on $n$ and $L$ and not on $\varphi$ or $z$. As in the proof of Theorem 6.10 we conclude that for all $x \in \mathbb{R}^{n-1}$ and $t>0$,

$$
\rho\left(\varphi\left(x+t e_{n}\right), \varphi(x)+t e_{n}\right) \leq C(n, L)+\log L=: \widetilde{C} .
$$

Now let $z_{1}=x_{1}+t_{1} e_{n}$ and $z_{2}=x_{2}+t_{2} e_{n}$, where $x_{1}, x_{2} \in \mathbb{R}^{n-1}$ and $t_{1}, t_{2}>0$. Then $\rho\left(z_{1}, z_{2}\right)=\rho\left(x_{1}-x_{2}+t_{1} e_{n}, t_{2} e_{n}\right)=\rho\left(\left|x_{1}-x_{2}\right|+t_{1} e_{n}, t_{2} e_{n}\right)$, and

$$
\begin{aligned}
\rho\left(\varphi\left(z_{1}\right), \varphi\left(z_{2}\right)\right) & \leq \rho\left(\varphi\left(x_{1}\right)+t_{1} e_{n}, \varphi\left(x_{2}\right)+t_{2} e_{n}\right)+2 \widetilde{C} \\
& =\rho\left(\left|\varphi\left(x_{1}\right)-\varphi\left(x_{2}\right)\right| e_{1}+t_{1} e_{n}, t_{2} e_{n}\right)+2 \widetilde{C} \\
& \leq \rho\left(L\left|x_{1}-x_{2}\right| e_{1}+t_{1} e_{n}, t_{2} e_{n}\right)+2 \widetilde{C} .
\end{aligned}
$$

Using the fact that $\mathbb{H}^{2}$ isometrically embeds in $\mathbb{H}^{n}$, we can explicitly compute these quantities and obtain $\rho\left(\varphi\left(z_{1}\right), \varphi\left(z_{2}\right)\right)-\rho\left(z_{1}, z_{2}\right) \leq 2 \log L+2 \widetilde{C}=: D$. Similarly, one obtains $\rho\left(\varphi\left(z_{1}\right), \varphi\left(z_{2}\right)\right)-\rho\left(z_{1}, z_{2}\right) \geq-2 \log L-2 \widetilde{C}=-D$, and this shows (9.6).

Now let $g=\varphi \gamma \varphi^{-1} \in G$, where $\gamma \in \Gamma$. Applying (9.6) to $\varphi$ and $\varphi^{-1}$ and using the invariance of the hyperbolic metric under Möbius transformations we obtain that for any $x, y \in \mathbb{H}^{n}$ we have

$$
|\rho(g(x), g(y))-\rho(x, y)| \leq 2 D .
$$

Since $\Gamma$ is convex co-compact, the series $\sum_{\gamma \in \Gamma} e^{-s \rho(y, \gamma(y))}$ diverges at the critical exponent $s=\delta$ for any $y \in \mathbb{H}^{n}$ (see [20]). Using (9.7) and the triangle inequality, one observes that the corresponding series for the group $G$ diverges as well. 
Thus we have for any $s>\delta, g \in G$ and $x, y \in \mathbb{H}^{n}$ :

$$
\begin{aligned}
\mu_{g^{-1}(x), y}^{s} & =\frac{1}{\sum_{h \in G} e^{-s \rho(y, h(y))}} \sum_{h \in G} e^{-s \rho\left(g^{-1}(x), h(y)\right)} \delta_{h(y)} \\
& \leq \frac{1}{\sum_{h \in G} e^{-s \rho(y, h(y))}} \cdot e^{2 s D} \sum_{h \in G} e^{-s \rho(x, g h(y))} \delta_{g^{-1} g h(y)} \\
& =e^{2 s D} g * \mu_{x, y}^{s} .
\end{aligned}
$$

A similar inequality holds in the other direction so that we obtain

$$
e^{-2 \delta D} \cdot \mu_{g^{-1}(x), y}^{\delta} \leq g * \mu_{x, y}^{\delta} \leq e^{2 \delta D} \cdot \mu_{g^{-1}(x), y}^{\delta} .
$$

This shows that assumption (9.1) holds for $G$.

The other assumption in Theorem 9.1 is satisfied for convex co-compact Kleinian groups and thus also holds for $G$, since the quasiconformal map $\varphi$ that conjugates $\Gamma$ to $G$ can only change a fixed angle by a bounded amount.

We finish with a conjecture. We have been unable to construct an example of a discrete QCF group $G$ so that $\delta(G)<\operatorname{dim}(L(G))$ and its Patterson-Sullivan measure is atom-free.

Conjecture 9.6. Let $G$ be a discrete $Q C F$ group acting on $\overline{\mathbb{B}^{n}}$, so that its resulting Patterson-Sullivan measures are atom-free. Then $\delta(G)=\operatorname{dim}(L(G))$.

Along these lines, we ask: If $G$ is convex co-compact and atom-free, then are the Patterson-Sullivan measures and the Hausdorff measure at the critical dimension comparable?

\section{ACKNOWLEDGEMENTS}

We would like to express our gratitude to Fred Gehring and Gaven Martin for their paper [8, which underlies much of the work in this paper. We also thank Fred Gehring, Juha Heinonen and Gaven Martin for stimulating discussions on the subject matter of this paper. In particular, we thank Gaven Martin for pointing out Lemma 3.5 to us.

\section{REFERENCES}

[1] J. Anderson, P. Bonfert-Taylor, and E. C. Taylor, Convergence groups, Hausdorff dimension, and a theorem of Sullivan and Tukia, preprint, 2002.

[2] A. F. Beardon, The geometry of discrete groups, Springer-Verlag, New York, 1983; corrected reprint, 1995. MR 85d:22026, MR 97d:22011

[3] C. J. Bishop and P. W. Jones, Hausdorff dimension and Kleinian groups, Acta Math. 179 (1997), 1-39. MR 98k:22043

[4] P. Bonfert-Taylor and G. Martin, Discrete quasiconformal groups of compact type, in preparation.

[5] P. Bonfert-Taylor and E. C. Taylor, Hausdorff dimension and limit sets of quasiconformal groups, Mich. Math. J. 49 (2001), 243-257. MR 2002g:30018

[6] B. Bowditch, Geometrical finiteness for hyperbolic groups, J. Funct. Anal. 113 (1993), 245317. MR 94e:57016

[7] M. H. Freedman and R. Skora, Strange actions of groups on spheres, J. Differential Geometry 25 (1987) 75-98. MR 88a:57074

[8] F. W. Gehring and G. J. Martin, Discrete quasiconformal groups I, Proc. London Math. Soc. (3) $\mathbf{5 5}$ (1987) 331-358. MR 88m:30057

[9] F. W. Gehring and G. J. Martin, Discrete quasiconformal groups II, unpublished manuscript. 
[10] M. Ghamsari, Quasiconformal groups acting on $B^{3}$ that are not quasiconformally conjugate to Möbius groups, Ann. Acad. Sci. Fenn. Ser. A I Math. (2) 20 (1995) 245-250. MR 96h:30034

[11] O. Lehto und K. I. Virtannan, Quasikonforme Abbildungen, Grundlehren der Mathematischen Wissenschaften, Springer-Verlag, 1965. MR 32:5872

[12] G. J. Martin, Discrete quasiconformal groups that are not the quasiconformal conjugates of Möbius groups, Ann. Acad. Sci. Fenn. Ser. A I Math. 11 (1986), 179-202. MR 89d:30025

[13] G. Martin, personal communication.

[14] B. Maskit, Kleinian groups, Springer-Verlag, 1988. MR 90a:30132

[15] V. Mayer, Cyclic parabolic quasiconformal groups that are not quasiconformal conjugates of Möbius groups, Ann. Acad. Sci. Fenn. Ser. A I Math. (1) 18 (1993), 147-154. MR 95f:30032

[16] P. J. Nicholls, The Ergodic Theory of Discrete Groups, Cambridge University Press, 1989. MR 91i:58104

[17] S. Patterson, The limit set of a Fuchsian group, Acta Math. 136 (1976), 241-273. MR 56:8841

[18] D. Stroock, Probability Theory, An Analytic View, Cambridge University Press, 1993. MR 95f:60003

[19] D. Sullivan, Hyperbolic geometry and homeomorphisms in geometric topology (Proc. Georgia Topology Conf., Athens, Ga., 1977), Academic Press, New York-London, 1979, 543-555. MR 81m:57012

[20] D. Sullivan, The density at infinity of a discrete group of hyperbolic motions, Publ. Math. IHES 50 (1979), 171-202. MR 81b:58031

[21] D. Sullivan, On the ergodic theory at infinity of an arbitrary discrete group of hyperbolic motions in Riemann surfaces and related topics (Proceedings of the 1978 Stony Brook Conference, State Univ. New York, Stony Brook, N.Y., 1978), Ann. of Math. Stud. 97, Princeton Univ. Press, Princeton, N.J., 1981, 465-496. MR 83f:58052

[22] D. Sullivan, Entropy Hausdorff measures old and new, and limit sets of geometrically finite Kleinian groups, Acta. Math. 153 (1984), pp. 259-277. MR 86c:58093

[23] P. Tukia, A quasiconformal group not isomorphic to a Möbius group, Ann. Acad. Sci. Fenn. Ser. AI Math. 6 (1981), 149-160. MR 83b:30019

[24] P. Tukia, The Hausdorff dimension of the limit set of a geometrically finite Kleinian group, Acta Math. 152 (1984), 127-134. MR 85m:30031

[25] P. Tukia, On quasiconformal groups, J. Analyse Math. 46 (1986), 318-346. MR 87m:30043

[26] P. Tukia, Conical limit points and uniform convergence groups, J. Reine Angew. Math. 501 (1998), 71-98. MR 2000b:30067

[27] P. Tukia and J. Väisälä, Quasiconformal extension from dimension $n$ to $n+1$, Ann. Math. 115 (1982), 331-348. MR 84i:30030

[28] J. Väisälä, Lectures on n-Dimensional Quasiconformal Mappings, Springer-Verlag, 1971. MR 56:12260

Department of Mathematics, Wesleyan University, Middletown, Connecticut 06459

E-mail address: pbonfert@wesleyan.edu

Department of Mathematics, Wesleyan University, Middletown, Connecticut 06459

E-mail address: ectaylor@wesleyan.edu 